Postprint version of article L.G. Barbu, A. Cornejo, X. Martínez, S. Oller, A.H. Barbat, Methodology for the analysis of post-tensioned structures using a constitutive serial-parallel rule of mixtures: Large scale non-linear analysis, Composite Structures 216C (2019) pp. 315-330, doi: 10.1016/j.compstruct.2019.02.092

\title{
METHODOLOGY FOR THE ANALYSIS OF POST-TENSIONED STRUCTURES USING A CONSTITUTIVE SERIAL-PARALLEL RULE OF MIXTURES: LARGE SCALE NON- LINEAR ANALYSIS
}

\author{
L.G.Barbu ${ }^{1,2}$, A. Cornejo ${ }^{1,2}$, X. Martínez ${ }^{1,2}$, S. Oller ${ }^{1,3}$ and A. H. Barbat ${ }^{1,2}$ \\ ${ }^{1}$ Centre Internacional de Mètodes Numèrics en Enginyeria (CIMNE), Campus Norte UPC, 08034 Barcelona, Spain \\ ${ }^{2}$ Universidad Politécnica de Cataluña (UPC), Campus Norte UPC, 08034 Barcelona, Spain \\ ${ }^{3}$ CONICET-UNSa (Consejo Nacional de Investigaciones Científicas y Técnicas), Salta, Argentina
}

\begin{abstract}
The main purpose of this paper is to develop a reliable method based on a three-dimensional (3D) finite-element (FE) model to simulate the constitutive behaviour of reinforced concrete structures strengthened with post-tensioned or pre-stressed tendons well beyond the elastic domain. The posttensioned concrete is modelled as a composite material whose behaviour is described with the serialparallel rule of mixtures (S-P RoM) [1-3] and the nonlinear behaviour of each component is accounted for by means of plasticity and damage models. 3D FE models were used, where the nonlinear material behaviour and geometrical analysis based on incremental-iterative load methods were adopted. Several examples are shown where the capabilities of the method on large scale structures are exhibited taking into account the self-weight, the post-tension load and different pressure loads. A new metric for computing the crack opening displacement inside a finite element is proposed.
\end{abstract}

Keywords: Pre-stressed, serial-parallel rule of mixtures, composites, failure analysis, plasticity, damage.

\section{Introduction and state of the art}

The work presented from here on is based on the innovative methodology proposed in [4] for the numerical analysis of pre-stressed and post-tensioned structures. In the aforementioned paper several different techniques have been mentioned as being habitually used when numerically calculating prestressed concrete structures.

In this paper the focus resides on these types of structures being taken well into the nonlinear domain due to failure either in the concrete or the steel tendons, or both. From this point of view, the model described by Rabczuk and Eibl [5] proposed a coupled element free Galerkin method to analyse prestrsessed concrete beams under quasi-static loading. The constitutive law governing the concrete medium was based on a coupled damage-plasticity model. The reinforcement was modelled as discrete beam elements so that the interaction between concrete and reinforcement can be modelled. The bond model included two modes of failure: pullout failure, and splitting failure. The formulation of the bond model was based on the radial stress-radial strain relation with three distinct domains: the nonlinear material behaviour including the initiation and propagation of cracks, linear softening, and residual strength.

More recently, Ayoub and Filippou proposed a nonlinear model for simulation of the pretensioned prestressed concrete girders [6]. The modelling approach consisted of three main components: concrete girder simulated as a beam-column, prestressing strands modelled as truss elements, and a bond element to model the prestress transfer between the concrete and strands. The constitutive laws governing the nonlinear response of concrete and strands were based on discretization of the media into fibers with uniaxial hysteric models. The bond model at the interface between the concrete and strand

\footnotetext{
${ }^{1}$ Corresponding author

e-mail address: lgratiela@cimne.upc.edu (L.G. Barbu)
} 
was formulated using special bond stress-slip relations.

Bílý and Kohoutková [7][8] conducted several studies on the effect of the steel liner on the ultimate bearing capacity of a prestressed concrete containment. Mechanical behaviour of concrete was modelled using a model that combines constitutive models for tensile (fracturing) and compressive (plastic) behaviour. The fracture model was based on the classical orthotropic smeared crack formulation and crack band model employing a Rankine failure criterion. The linear elements representing the tendons and the bars were embedded in concrete. Perfect bond between the concrete and these elements was considered.

The previous method can describe the behaviour of the composite material but, implicitly, the finite element mesh is strongly dependent on the trajectory of the steel tendons since they connect pair of nodes of the finite element. This means that in zones with high curvatures of the tendons a high density of finite elements has to be used, with the consequent increase of the computational cost. In addition, the friction or debonding effects between the concrete and steel cannot be easily included.

Alternatively, the formulation proposed in this article only requires a finite element mesh of any type and any spatial discretization and a linear description of the steel tendons. The pre-tensioned concrete is modelled as a composite material whose behaviour is described with the serial-parallel rule of mixtures (S/P RoM). The effective pre-tensioning stress is applied as an initial strain imposition only in the steel material used to model the tendons [9]. By means of the S-P RoM [1,2] equilibrium is reached at each integration point between the passive and active steel and concrete and the strain tensor of the steel is updated with the contribution of the concrete. An important advantage of applying the proposed methodology is that one can assume that each material behaves following its own constitutive law (elasticity, damage, plasticity, viscoelasticity, etc.). Next, the displacement field is updated until the global convergence of forces is achieved. The methodology is valid for both straight and curvilinear steel tendons and examples of both cases will be shown.

For the concrete an isotropic scalar damage model has been used together with a new metric for computing the crack opening displacement inside a finite element that the authors propose for the first time in this work. The model is described in detail in Section 3.1. The post-tensioned steel is modelled with an isotropic hardening-softening plasticity law, described in detail in Section 3.2.

Regarding the actual strain imposed on the steel material in those elements that contain a steel tendon, the losses due to friction induce a variation of the post-tensing force along the longitudinal axis of the tendon. In the proposed formulation, constant stress distributions is applied. In order to overcome this discrepancy, the force distribution obtained from the regulatory guide [10] can be integrated obtaining an "average" force, which is the one that should be imposed in the active steel of the composite material. For this, the averaged stress is divided by the Young modulus of the material obtaining therefore the imposed post-tensing strain.

\section{Constitutive modelling of pre-stressed reinforced concrete with the Serial-Parallel rule of mix- tures}

The Serial-Parallel rule of mixtures (S-P RoM) defines two different compatibility conditions between the strains and stress states of the composite constituent materials: it formulates an iso-strain condition on the parallel direction, usually the fibre direction, and it defines an iso-stress condition on the serial direction, usually the remaining directions. Using these compatibility equations in a composite made of matrix and fibre, if the matrix structural capacity is lost due to excessive shear stresses, the isostress condition also reduces the shear capacity of fibre, and, consequently, the composite serial strength is also reduced.

The extended formulation for the S-P RoM can be found in [4], together with its algorithm. It is interesting to summarize here the main hypothesis for the numerical formulation:

1. The composite is composed by only two components: fibre and matrix

2. Component materials have the same strain in parallel (fibre) direction.

3. Component materials have the same stress in serial direction. 
4. Composite material response is in direct relation with the volume fractions of compounding materials.

5. Homogeneous distribution of phases is considered in the composite.

6. Perfect bounding between components is considered.

\subsection{Singularities of the imposed strain procedure}

The previous numerical formulation is capable of simulating the behaviour of reinforced concrete as a composite material composed by concrete and passive steel. If one wants to take into account the case of the active steel, both for the pre and the post-tensioned case, it is necessary to rewrite the compatibility condition of the S-P RoM. Essentially, the perfect adherence between the two materials cannot be achieved since the compatibility is not ensured.

Referring to the hypothesis 6 of the S-P RoM, now it must be rewritten as: Relative movement between the components is allowed if and only if an imposed strain condition exists over one of them.

Therefore, loss of adherence is allowed only in the presence of the imposed strain loading, a peculiar load due to the fact that it is applied only on a component of the composite material. This implies that its contribution is not quantified in the external forces vector and it is an auto balanced load.

In the first iteration of the S-P RoM algorithm, the parallel component of the strain tensor of the active steel is fixed to the imposed pre-stress value. Knowing the composite stress tensor and the parallel component of the strain of the fibre, one can obtain the total strain tensor of the fibre and, depending on the desired fibre constitutive model, the integrated stress tensor is computed.

The algorithm of the S-P RoM equilibrates the serial components at each integration point and with the integrated stresses the internal forces vector is assembled. At this point of the problem, the parallel component of the fibre stresses has still to be balanced. Its effect is quantified in the system of equations in the residual forces computed at the end of the first global iteration of the problem.

$$
\begin{aligned}
& { }^{m} \varepsilon_{P}={ }^{c} \varepsilon_{P} \\
& \text { incr }=1 \text {; iter }=1 ; \quad{ }^{f} \varepsilon_{P}=\varepsilon_{\text {imp }} \\
& { }^{m} \varepsilon_{P}={ }^{c} \varepsilon_{P} \\
& \text { incr } \geq 1 \text {; iter }>1 ; \quad \quad{ }^{f} \varepsilon_{P}={ }^{c} \varepsilon_{P}+\varepsilon_{\text {imp }}
\end{aligned}
$$

The residual forces computed from the unbalanced internal forces vector are used to update the nodal displacements and the composite strains at each integration point. In the second global iteration of the structure, the parallel component of the strains at layer level (that is the input of the S-P RoM) is equal to the matrix strain needed to accommodate the imposed fibre strain.

Therefore, in the first global iteration the active steel has its parallel strain component fixed at the level of strain associated to the desired pre-stressing force, while in the second iteration the fibre strain is balanced by the resulting compression in the concrete.

\subsection{Differences between numerical modelling of pre-stressed and post-tensioned concrete.}

The two different types of enhancing the stiffness and strength of concrete can each be taken into account by the proposed methodology. The post-tensioning of steel is modelled by considering the three transversal modulus of the steel close to 0 and therefore allowing the steel to glide without friction inside the concrete. In order to fully allow deformation of the steel in a decoupled way from the concrete, its Poisson coefficients are set to 0 , which allows the complete description of the post-tensioning process.

Pre-stressing, on the other hand, is reproduced by considering both the transversal modulus and the 
Poisson coefficients for steel at their habitual value found in literature.

For both procedures, the elements that have a determined participation of active steel have a linear elastic behaviour for the concrete that belongs to that same layer as the active steel. This is necessary due to the very high value of the pre-stressing load. If a damage behaviour were to be assumed for the concrete in those same elements, then the concrete material would be fully damaged only by applying the pre/post-tension load which would difficult the convergence in the Newton -Raphson algorithm of the S-P RoM. While this is a natural consequence of the pre-stressing procedure, in the numerical analysis, global convergence of the problem would be difficult to achieve due to this highly localized effect and therefore the non-linear behaviour of real-life structures would be impossible to capture.

The authors consider this a reasonable simplification that enables us to follow the large-scale behaviour of structures well past the elastic domain.

\section{Component constitutive models}

\subsection{Isotropic damage model}

During the last years, the constitutive models known as continuous damage models have been widely accepted for the simulation of the complex constitutive behavior of many materials used in engineering.

Kachanov [11] introduced the concept of effective stresses to carry out fracture simulations by viscous phenomena. It is also used for the representation of fatigue, fracture in ductile and fragile materials, etc. [12].

The isotropic damage model is widely known and therefore only some brief explanations will be given from here on. The readers can find the complete formulation in references [13][14].

For the isotropic damage model, the material degradation is developed in all directions alike and only depends on one scalar damage variable $d$, and the equation of isotropic damage is then

$$
\sigma_{0}=\frac{\sigma}{1-d}
$$

Where $d$ is the internal damage variable, $\sigma$ is Cauchy's stress tensor and $\sigma_{0}$ is the effective Cauchy's stress tensor measured in the "non-damaged" space. The relation between the real damaged space and the non-damaged one can be seen in a schematic way in Figure 1. This internal variable is a measure of the material stiffness loss and its higher or lower limits are given by $0 \leq d \leq 1$ where $d=1$ is a state of the material completely damaged that defines the complete local fracture, and $d=0$ is a nondamaged material.

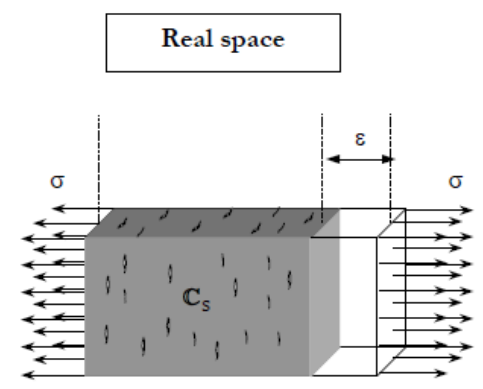

a) Damaged real solid

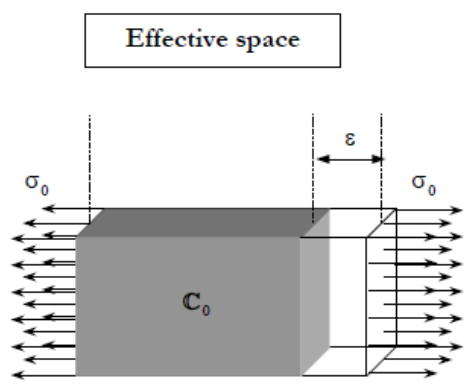

b) Non-damaged equivalent solid

Figure 1. Schematic representation of the equivalence between the real and undamaged space

\subsubsection{Damage threshold criterion}

The damage criterion makes a distinction between an elastic behavior inside the domain delimited by the damage function and another domain in which the degradation process of the material properties is verified. This criterion depends on the type of material and is defined in the same way as for plasticity problems, 


$$
\mathrm{F}\left(\boldsymbol{\sigma}_{\mathbf{0}} ; \boldsymbol{q}\right)=f\left(\boldsymbol{\sigma}_{\mathbf{0}}\right)-c(d), \text { with } \boldsymbol{q} \equiv d
$$

where $f\left(\boldsymbol{\sigma}_{\mathbf{0}}\right)$ is a function of the stress tensor $\boldsymbol{\sigma}_{\mathbf{0}}=\boldsymbol{C}_{\boldsymbol{o}}: \boldsymbol{\varepsilon}$ and $c(d)$ is the function defining the damage threshold position. This function establishes the onset of the nonlinear damage behavior and additionally defines the loading, unloading and reloading states. The initial value of the damage threshold $c\left(d^{0}\right)=c^{\max }=\sigma^{\max }$ is a property of the material and is related to its strength in compression depending on the damage threshold function chosen. The damage in the material is verified when the value of $f\left(\boldsymbol{\sigma}_{\mathbf{0}}\right)$ is equal or greater than $c^{\max }=\sigma^{\max }$ for the first time.

\subsubsection{Evolution law of the internal damage variable}

The scalar function $G[\chi]$ defining the evolution of the damage threshold must be monotonous and with a value ranging from 0 to 1 . In various publications about the scalar damage problem, the stress behaviour with softening is represented in a variety of forms. Particularly, in Oliver's et al. [13] work the following function is proposed,

$$
G(c(d))=1-\frac{c^{\max }}{c(d)} e^{A\left(1-\frac{c(d)}{c^{\max }}\right)}
$$

Or it can be expressed as,

$$
G\left(f\left(\boldsymbol{\sigma}_{\mathbf{0}}\right)\right)=1-\frac{f^{0}\left(\boldsymbol{\sigma}_{\mathbf{0}}\right)}{f\left(\boldsymbol{\sigma}_{\mathbf{0}}\right)} e^{A\left(1-\frac{f\left(\boldsymbol{\sigma}_{\mathbf{0}}\right)}{f^{0}\left(\boldsymbol{\sigma}_{\mathbf{0}}\right)}\right)}
$$

where $A$ is a parameter that depends on the fracture energy of the material. In the following table, the algorithm of this already integrated model is presented. It is easier to use but less general because only exponential softening is used.

1. Calculation of the predictive stress and internal damage variable for the current time " $t+\Delta t$ ", equilibrium iteration " $i$ ",

$$
\begin{aligned}
& {\left[\boldsymbol{\sigma}_{0}\right]^{t+\Delta t}=\mathbb{C}_{0}:[\boldsymbol{\varepsilon}]^{t+\Delta t}} \\
& { }^{i}[d]^{t+\Delta t} ; \tau={ }^{i}\left[G\left[f\left(\boldsymbol{\sigma}_{0}\right)\right]^{t+\Delta t}\right.
\end{aligned}
$$

2. Verification of the damage threshold condition:

a. If: $\tau-\tau^{\max } \leq 0$

$$
\text { Then }\left\{\begin{array}{l}
{ }^{i}[\boldsymbol{\sigma}]^{t+\Delta t}=\left[\boldsymbol{\sigma}_{0}\right]^{t+\Delta t} \\
{ }^{i}[d]^{t+\Delta t} ; \tau^{\max }=\tau
\end{array}\right\} \text { and goes to the EXIT }
$$

b. If: $\tau-\tau^{\max }>0$ Then starts the integration of the constitutive equation.

3. Integration of the equation itself,

$$
\begin{aligned}
& \tau^{\max }=\tau \\
& { }^{i}[d]^{t+\Delta t}=1-\left[\frac{f^{0}\left(\boldsymbol{\sigma}_{0}\right)}{\tau} \mathrm{e}^{A\left(1-\frac{\tau}{f^{0}\left(\sigma_{0}\right)}\right)}\right]^{t+\Delta t}
\end{aligned}
$$

4. Update the stress and the tangent constitutive tensor.

$$
\begin{aligned}
& { }^{i}[\boldsymbol{\sigma}]^{t+\Delta t}=\left(1-{ }^{i}[d]^{t+\Delta t}\right)\left[\boldsymbol{\sigma}_{0}\right]^{t+\Delta t} \\
& { }^{i}\left[\mathbb{C}^{T}\right]^{t+\Delta t}=\left[(1-d) \mathbb{C}_{0}-\frac{\partial G\left[f\left(\boldsymbol{\sigma}_{0}\right)\right]}{\partial\left[f\left(\boldsymbol{\sigma}_{0}\right)\right]}\left[\mathbb{C}_{0}: \boldsymbol{\varepsilon}\right] \otimes\left[\frac{\partial f\left(\mathbb{C}_{0}: \boldsymbol{\varepsilon}\right)}{\partial \boldsymbol{\varepsilon}}\right]\right]^{t+\Delta t}
\end{aligned}
$$

Table 1. Algorithm for obtaining the stress of the damage model 


\subsubsection{Evaluation of the crack opening}

The scalar damage model computes a damage variable that quantifies the damaged volumetric percentage of the finite element. However, this variable does not take into account the effect caused by the opening and closing of cracks.

In order to take this into consideration a crack displacement is computed inside the finite element code at each integration point as:

$$
u_{c r}=\left(d \cdot \varepsilon_{e q}\right) \cdot l_{f}
$$

Where $\varepsilon_{e q}$ is the equivalent strain of the Gauss point and $l_{f}$ is the characteristic length of the element.

The equivalent strain is computed as:

$$
\varepsilon_{e q}=\frac{\boldsymbol{\sigma}: \boldsymbol{\varepsilon}}{f(\boldsymbol{\sigma})}
$$

Where $f(\boldsymbol{\sigma})$ is the uniaxial equivalent stress. This ensures that when an element is unloaded its crack displacement can tend to 0 (that crack is closing), even though in that material volume, degradation has been previously recorded $(d>0)$.

\subsection{Plasticity model}

The limit between the elastic and the plastic areas is set through the yield surface or discontinuity surface, and from such limit, this surface can move in the stress space, follow the evolution of the plastic process and transform itself into the so called plastic loading surface. This function representing the plastic loading surface is simply the discontinuity or yield limit function updated for each of the internal variable value $\mathbf{q}$ at every moment of the pseudo time $t$ of the elasto-plastic process. The phenomenon governing this yield surface change of position in the stress space is known as plastic hardening. This hardening behavior in this case is isotropic or kinematic and will be presented below.

A simple way of introducing the elasto-plastic behavior hardening is through the plastic loading function $\mathrm{F}(\boldsymbol{\sigma} ; \boldsymbol{q})=0$. This is defined as a scalar tensor and homogenous function of first degree in the stresses.

$$
\mathrm{F}(\boldsymbol{\sigma} ; \boldsymbol{q})=f(\boldsymbol{\sigma})-K=0
$$

Thus, the stress function $f(\boldsymbol{\sigma})$ is set to translate from a stress tensor state into another equivalent scalar. This scalar is compared to the evolution of the plastic hardening $K$, which is related to the evolution of the equivalent uniaxial stress.

\subsubsection{Isotropic hardening}

It is said that there is an isotropic hardening when there is a homothetic movement of the plastic loading surface. This movement can be:

- Positive: when the homothetic movement of the plastic loading surface is an expansion movement; it is said that it is an isotropic hardening elasto-plastic process.

- Null: when there is no evolution of the plastic loading surface during the elasto-plastic process, it is said that this is an isotropic perfectly elasto-plastic process.

- Negative: when there is a contraction homothetic movement on the plastic loading surface, it is said that this is an isotropic softening elasto-plastic process

The isotropic hardening, the homothetic movement of the plastic loading function, is controlled by the evolution of the plastic hardening function $K$, which is generally defined as an internal variable $\mathbf{q}$. The evolution of this internal variable depends on the mechanical process. 
This internal variable evolution depends on its own mechanical process and the latter is conditioned by an evolution rule whose formulation must adapt itself to the solid behaviour. In the classic plasticity, the internal variable of the plastic hardening is usually expressed as a plastic hardening function $K\left(\kappa^{p}\right)$, which, in turn, depends on the internal variable of plastic hardening $\kappa^{p}$.

By defining the hardening function as an internal variable of the plastic process, a more general formulation arises, so there are greater possibilities to represent the behaviour of a large variety of solids.

$$
\begin{gathered}
\dot{\kappa}^{p}=\dot{\lambda} H_{k}(\boldsymbol{\sigma} ; \boldsymbol{q})=\dot{\lambda}\left[\boldsymbol{h}_{k}(\boldsymbol{\sigma} ; \boldsymbol{q}): \frac{\partial G}{\partial \boldsymbol{\sigma}}\right] \\
\dot{K}^{p}=\dot{\lambda} H_{k}(\boldsymbol{\sigma} ; \boldsymbol{q})=\dot{\lambda}\left[h_{k}(\boldsymbol{\sigma} ; \boldsymbol{q}) \dot{\kappa}^{p}\right]
\end{gathered}
$$

where the tensor function $\boldsymbol{h}_{k}(\boldsymbol{\sigma} ; \boldsymbol{q})$ and the scalar function $h_{k}(\boldsymbol{\sigma} ; \boldsymbol{q})$ depend on the updated tensor state and the internal variables.

In the Barcelona model defined in Lubliner et al.[15], the laws defined are driven by the fracture energy of the material. This work presents a new law that has been designed to reproduce their hardening and softening performance under monotonic and cyclic loading conditions. This law also depends on the fracture energy of the material and is derived from the hardening softening law presented in [16] and [17].

\subsubsection{Fracture Energy}

Classical fracture mechanics defines the fracture energy of a material as the energy that has to be dissipated to open a fracture in a unitary area of the material. This energy is defined as:

$$
G_{f}=\frac{W_{f}}{A_{f}}
$$

where $W_{f}$ is the energy dissipated by the fracture at the end of the process, and $A_{f}$ is the area of the surface fractured. The total fracture energy dissipated, $W_{f}$, in the fracture process can be used to define a fracture energy by unit volume, $g_{f}$, required in a continuum mechanics formulation:

$$
W_{f}=G_{f} \cdot A_{f} \equiv \int_{V_{f}} g_{f} d V
$$

This last equation allows establishing the relation between the fracture energy defined as a material property, $G_{f}$, and the maximum energy per unit volume $g_{f}$ :

$$
g_{f}=\frac{W_{f}}{V_{f}}=\frac{W_{f}}{A_{f} \cdot l_{f}}=\frac{G_{f}}{l_{f}}
$$

Where $W_{f}$ is the total fracture energy dissipated, $V_{f}$ is the volume of the finite element, $A_{f}$ is the area of the surface fractured and $l_{f}$ is the characteristic finite element length.

Thus, the fracture energy per unit volume is obtained as the fracture energy of the material divided by the fracture length. This fracture length corresponds to the distance, perpendicular to the fracture area, in which this fracture propagates. 
In a real section, this length tends to be infinitesimal. However, in a finite element simulation, in which continuum mechanics is applied to a discrete medium, this length corresponds to the smallest value in which the structure is discretized: the length represented by a gauss point.

Therefore, in order to have a finite element formulation consistent and mesh independent, it is necessary to define the hardening law in function of the fracture energy per unit volume [15]. This value is obtained from the fracture energy of the material, $G_{f}$, and the size of the finite element in which the structure is discretized.

\subsubsection{Hardening Function and Hardening Internal Variable}

The hardening function defines the stress of the material when it is in the non-linear range. There are many possible definitions that can be used to fulfil the rate equations for the plastic strength threshold. Here the use of a function that describes the evolution of an equivalent uniaxial stress state is proposed, like the one shown in Figure 2.

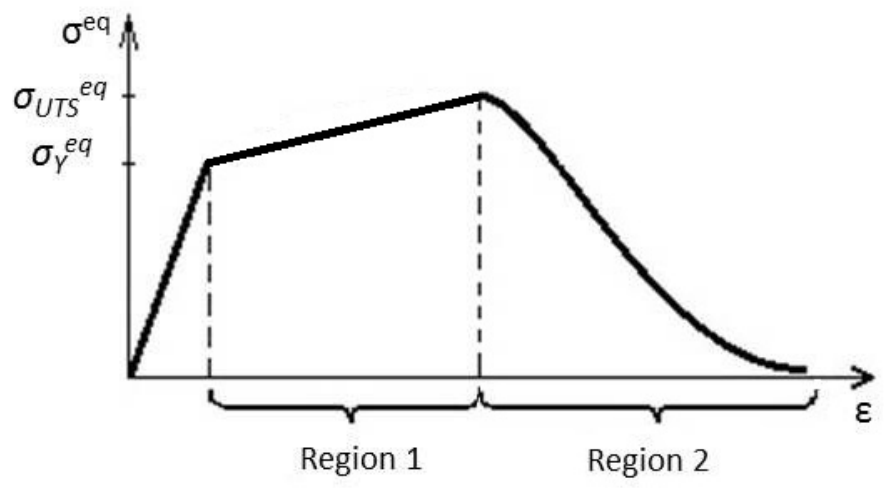

Figure 2. Evolution of the equivalent stress

The equivalent stress state shown in Figure 2 has been defined to match the uniaxial stress evolution described by most metallic materials.

The first region is defined as a linear curve. The second region is defined with an exponential function to simulate softening. The function starts with a null slope that becomes negative as the equivalent plastic strains increase. The exact geometry of this last region depends on the fracture energy of the material.

The hardening internal variable, $\kappa^{p}$, accounts for the evolution of the plastic hardening function, $K$. In current formulation $\kappa^{p}$ is defined as a normalized scalar parameter that takes into account the amount of volumetric fracture energy dissipated by the material in the actual strain-stress state. This is:

$$
\kappa^{p}=\frac{1}{g_{f}} \int_{t=0}^{t} S: \dot{E}^{p} d t
$$

Where $g_{f}$ is the total available energy per unit volume, $S$ is the stress tensor and $E^{p}$ is the rate of plastic strain.

Using the definition of the hardening internal variable defined in equation (13), it is possible to define the expression of the hardening function as:

$$
K=S^{e q}\left(\kappa^{p}\right)
$$

Where $S^{e q}$ is the equivalent stress tensor whose expression depends on the region of the constitutive law. It can be easily proven that the hardening function and internal variable defined in equations (13) 
and (14) fulfil the rate equations (9) and (10). And the $h_{k}$ and $h_{\mathbf{k}}$ functions defined in this expression become:

$$
\begin{aligned}
& h_{k}=\frac{\partial S^{e q}}{\partial \kappa^{p}} \\
& h_{\mathrm{k}}=\frac{S}{g_{t}}
\end{aligned}
$$

\subsubsection{Expressions of the hardening function}

In this section, the exact numerical expressions used to define the new hardening law are discussed. This law is a simplified version of the one presented in Barbu et al [17].

\section{Region 1: Linear curve}

The threshold function is obtained taking into account the following considerations:

- The initial equivalent stress value is defined by the equivalent stress reached at the end of the elastic domain, the material strength $\sigma_{\text {lim,el }}$.

- The slope of the function, $u$, is user defined, $u=\frac{\sigma_{1}^{e q}-\sigma_{\text {lim,el }}}{\varepsilon_{1}^{p}}$.

- The volumetric fracture energy dissipated in this region is $g_{t 1}=\left(\sigma_{1}^{e q}+\sigma_{l i m, e l}\right) \cdot \varepsilon_{1}^{p} \cdot 0.5$.

With these considerations in mind, the resulting equation that relates the equivalent stress with the plastic strain is:

$$
\sigma^{e q}\left(\varepsilon^{p}\right)=\sigma_{\text {lim,el }}+u \cdot \varepsilon_{p}
$$

The expression of the equivalent stress as a function of the hardening variable is obtained:

$$
\sigma^{e q}\left(k^{p}\right)=\sqrt{\sigma_{l i m, e l}^{2}+2 \cdot u \cdot g_{t} \cdot k^{p}}
$$

Expression (18) is valid for values of $\kappa^{p}$ that are comprehended between 0 and $k_{1}^{p}=g_{t 1} / g_{t}$. The value of the upper limit of the internal variable shows that it is necessary define a value for the volumetric fracture energy of the material larger than $g_{t 1}$. If the value defined is lower, the material will not be able to reach its ultimate stress as this will imply having a fracture internal variable larger than 1.0 .

\section{Region 2: Exponential softening}

When the plastic internal variable reaches the volumetric plastic energy available in the first region: $k^{p}=k_{1}^{p}$. At this point, isotropic hardening is defined by region two. Its function is obtained with the following parameters:

- The initial equivalent stress value is defined by the equivalent stress reached at the end of the first region $\left(\sigma_{1}^{e q}\right)$.

- The initial slope of the function is zero.

- The volumetric fracture energy dissipated in this region is the remaining energy in the material: $g_{t 2}=g_{t}-g_{t 1}$ 
With these considerations in mind, the governing equations are the same as in Barbu et al [17]. The constitutive laws described in this section have been implemented in the in-house code PLCd [18]. The code was programmed to allow OpenMP parallelization, which greatly reduced the computational cost of the large scale FE simulations.

\section{Large scale non-linear analysis of pre-stressed and post-tensioned structures}

In this section, two different large-scale structures that have steel tendons embedded in the concrete structure can be seen. Their elastic behaviour has been shown in [4] and here the nonlinear behaviour will be presented. For the steel tendons, a plasticity model with isotropic hardening has been used while for the concrete an isotropic scalar damage model was chosen. Both structures presented in this section have three different loading cases. First, the self-weight load is applied. Afterwards, the pre/post-tensioning load is applied and finally a distributed load is applied monotonically until the structure is considered to have failed completely. A component is considered to have failed when either its damage variable has reached a level of 0.9 or more (in the case of concrete), or its normalized plastic dissipation has reached a similar value for the steel.

\subsection{Linear beam with two linear steel tendons}

In this case, a linear $1.5 \mathrm{~m}$ length beam with a typical industrial cross section has been analysed. There are two linear steel tendons embedded in the concrete pre-stressed up to $1092.84 \mathrm{MPa}$ which corresponds to a strain equal to 0.0056 . The Young modulus of the steel has been considered equal to $195150 \mathrm{MPa}$ and for the concrete is equal to $40200 \mathrm{MPa}$. The Poisson ratio is equal to 0.3 for the steel and 0.2 for the concrete.

The geometry of the beam and its cross section can be seen in the Figs. 3 and 4 .

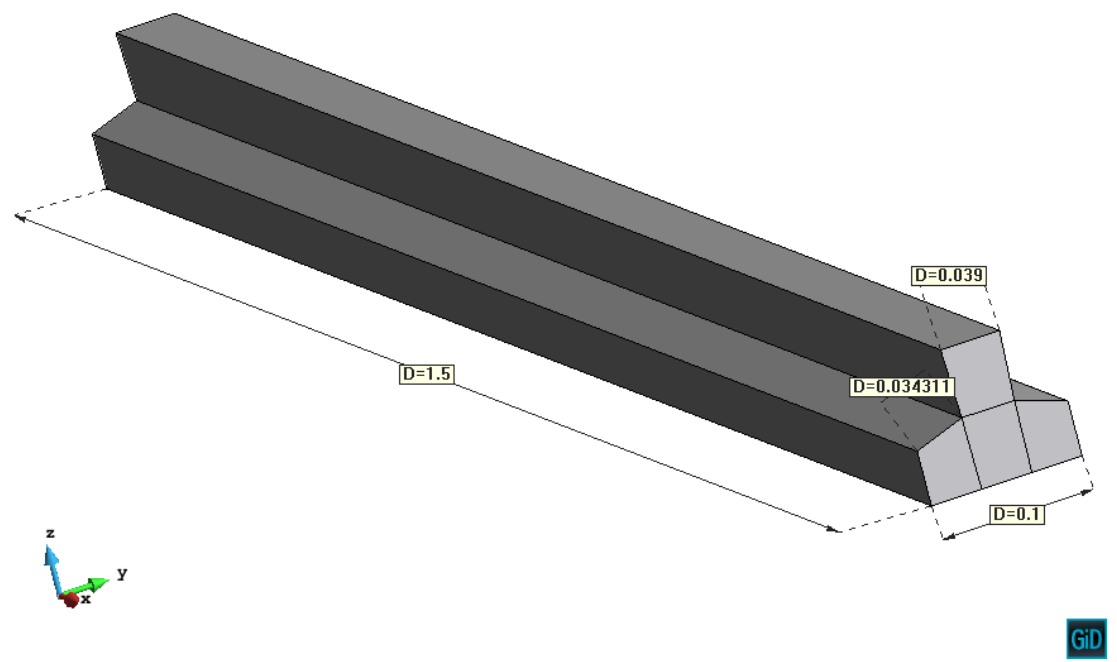

Figure 3. Geometry of the beam, dimensions in $\mathrm{m}$.

Concrete has been modelled with the constitutive model described in Section 3, using the MohrCoulomb failure surface described in [14] and an exponential softening law. The compressive strength of concrete is $48.7 \mathrm{MPa}$ and the scaling factor between the compressive and tensile strength is 11.2, leading to a tensile strength of 4,34MPa. The tensile fracture energy considered for concrete is $400 \mathrm{MN} / \mathrm{m}$. For steel, an elastic limit of 1000MPa has been considered together with a Von Mises yield surface and a Von Mises potential criterion. The fracture energy considered is $1000 \mathrm{~N} / \mathrm{m}$ and the plastic strain at which softening is set to start is 0.001 . This parameter is necessary for the constitutive law, as explained in Section 3. 


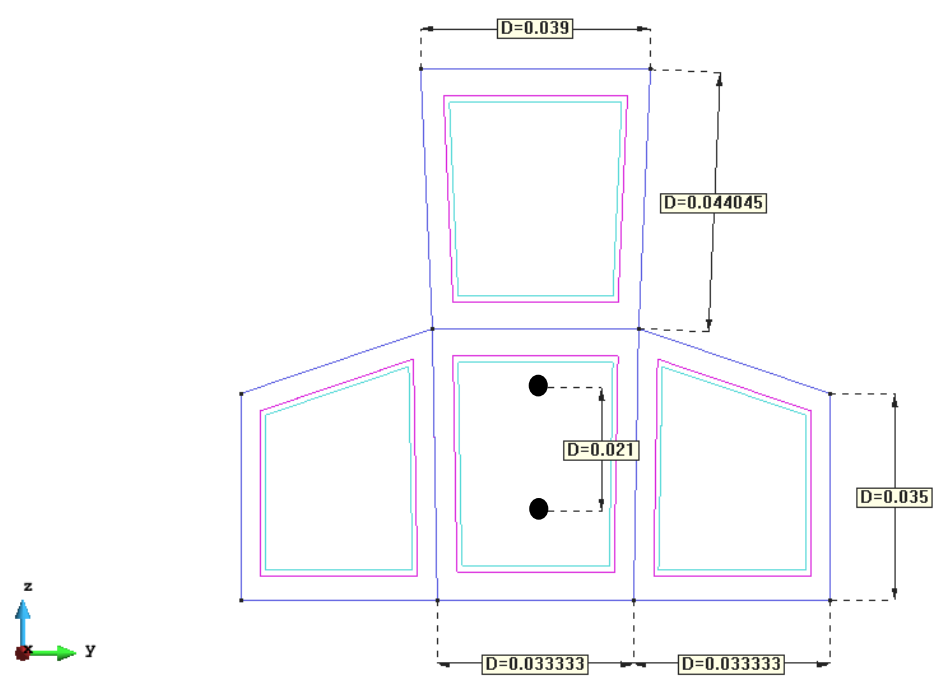

Figure 4. Cross section of the beam, dimensions in $\mathrm{m}$.

The structured finite element mesh can be seen in the Fig. 5. This mesh is composed of 12750 linear hexahedral finite elements and 16308 nodes. The finite elements intersected by the steel tendons can be seen in Fig.6. For this simple case, only two composite materials were generated, since the entrance angle and volumetric participation along the finite elements intersected by each tendon is constant. This means that each finite element intersected by the steel tendon has a certain volumetric participation of active steel, on which the imposed strain procedure (see section 2.1) is going to be applied.

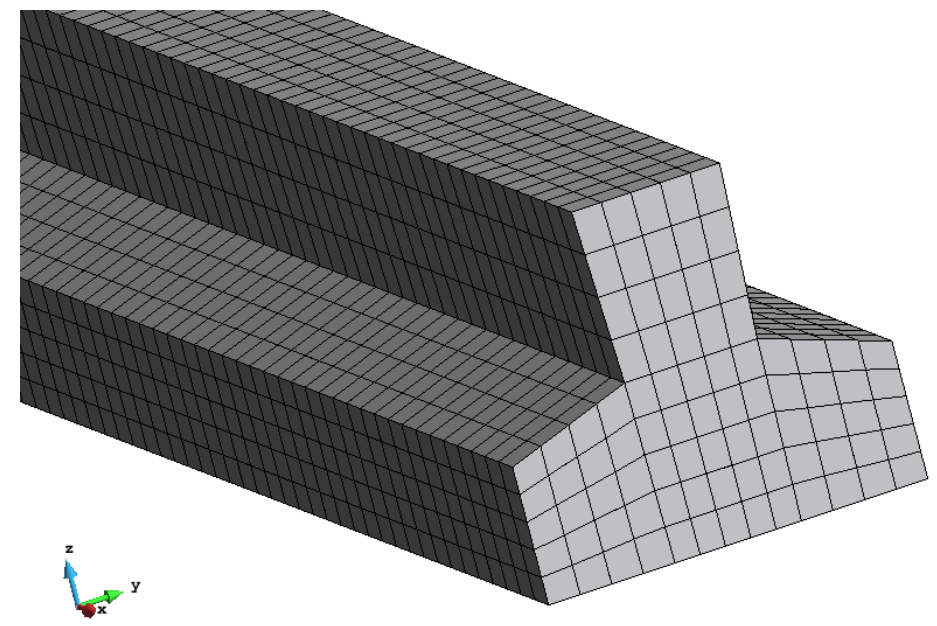

Figure 5. Finite element mesh used (12750 finite elements) 


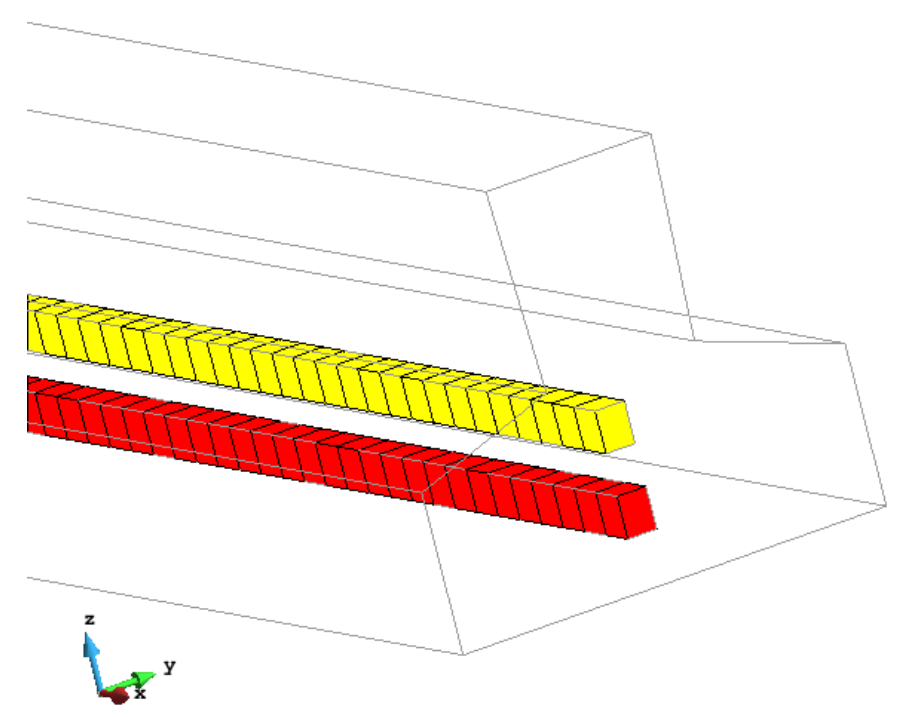

GiD

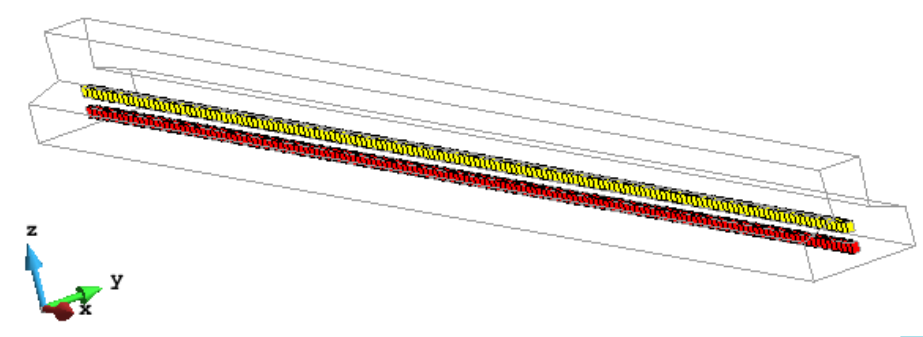

Figure 6. Finite elements intersected by the steel tendon

First, the self weight load has been applied. To demonstrate the versatility of the pre-stressing procedure only one of the steel tendons has been pre-stressed. The strain imposed was 0.0056 for the active steel contained in those finite elements that were intersected by the first tendon. Afterwards, a pressure of 0.535 bars has been applied incrementally.

In Figure 7, the displacement field as resulting from the self-weight load can be seen, while in the figures 8 to 11 the structure is described after the pre-stress load has been applied. It can be seen by comparing Figures 7 and 8 that the pre-stressing of the steel tendons compensates the curvature induced by the self-weight loading.

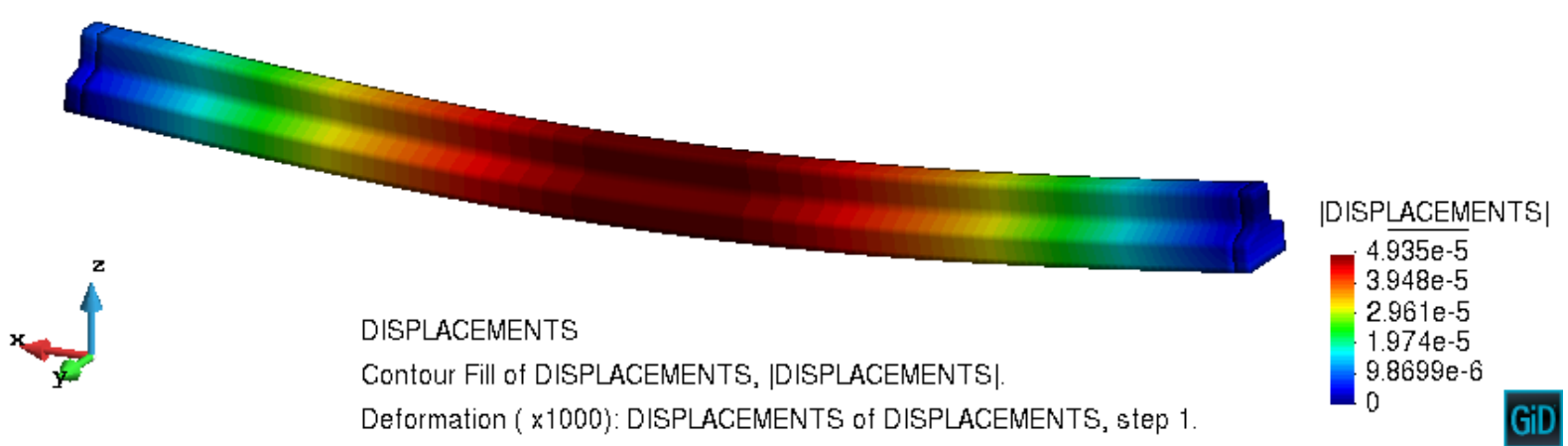

Figure 7. Displacement field after self-weight, shown on the deformed shape (x1000) 


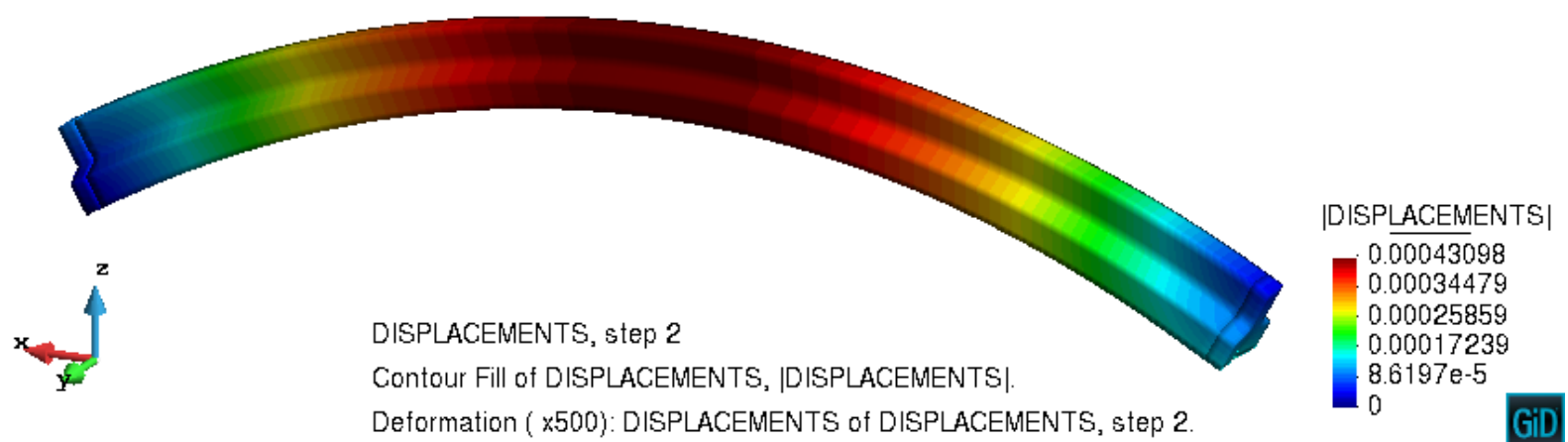

Figure 8. Displacement field at the end of the pre-stress load, shown on the deformed shape (x500)

Figures 9 and 10 show the distribution of the plasticity and damage internal variables in the steel and concrete, respectively. Both results are shown on the deformed shape of the beam with a scaling factor of 500. It can be seen that at the end of the pre-stressing stage the steel has dissipated at its most tensioned point $36.8 \%$ of its internal energy. Concrete, on the other hand, exhibits degradation in those elements that are closest to the pre-stressed steel tendon, as can be seen in Figure 10. This is to be expected, due to the high strains introduced by the pre-stressing of the steel tendon and due to the perfect adherence considered between the two materials.

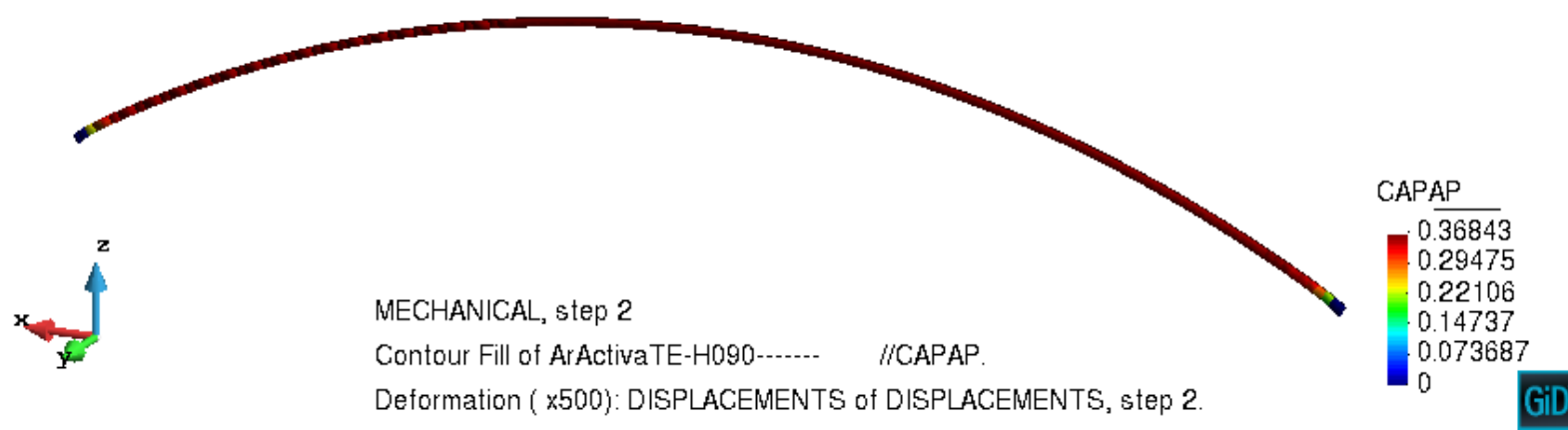

Figure 9. Normalized plastic dissipation for the steel at the end of the pre-stressing load (CAPAP $=\kappa^{p}$ )

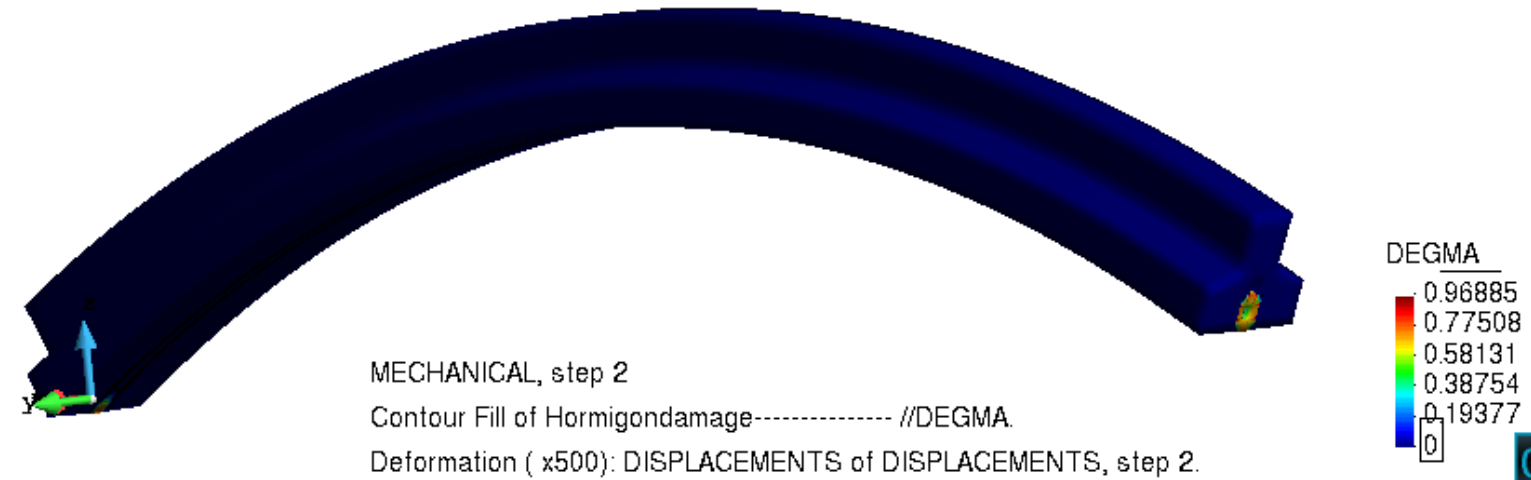

Figure 10. Damage distribution in the concrete at the end of the pre-stressing load $(\mathrm{DEGMA}=d$ ) 


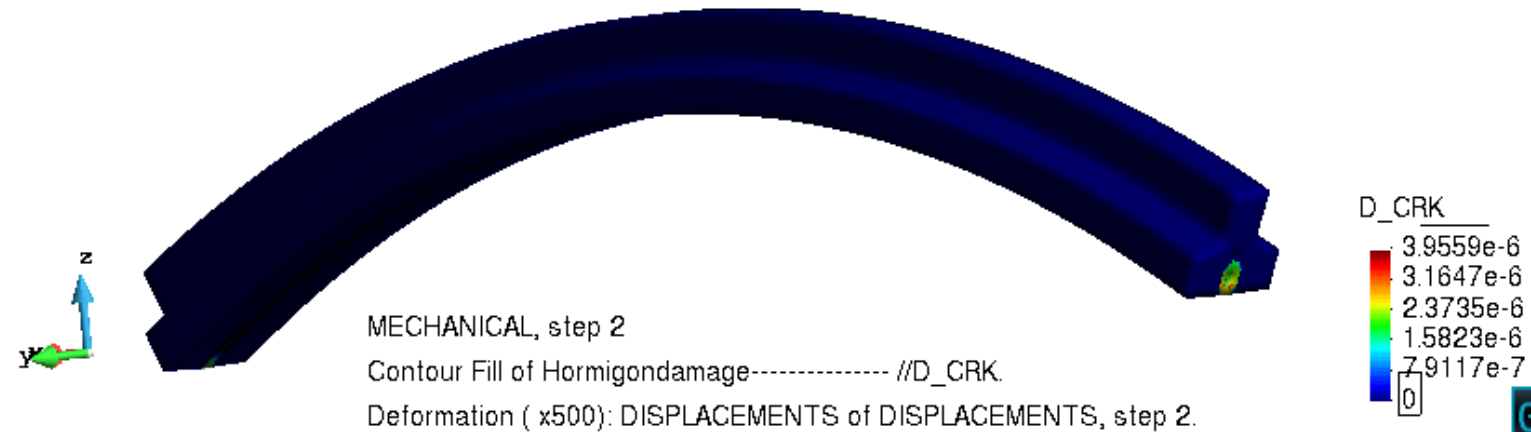

Figure 11. Crack opening distribution in the concrete at the end of the pre-stressing load (m)

Figure 11 shows that micro cracks have appeared in the concrete with a maximum opening of $0.0039 \mathrm{~mm}$. These cracks are situated in the closest elements that are aligned to the trajectory of the steel tendon. The crack opening has been calculated for each finite element according to the procedure described in Section 3.1.3.

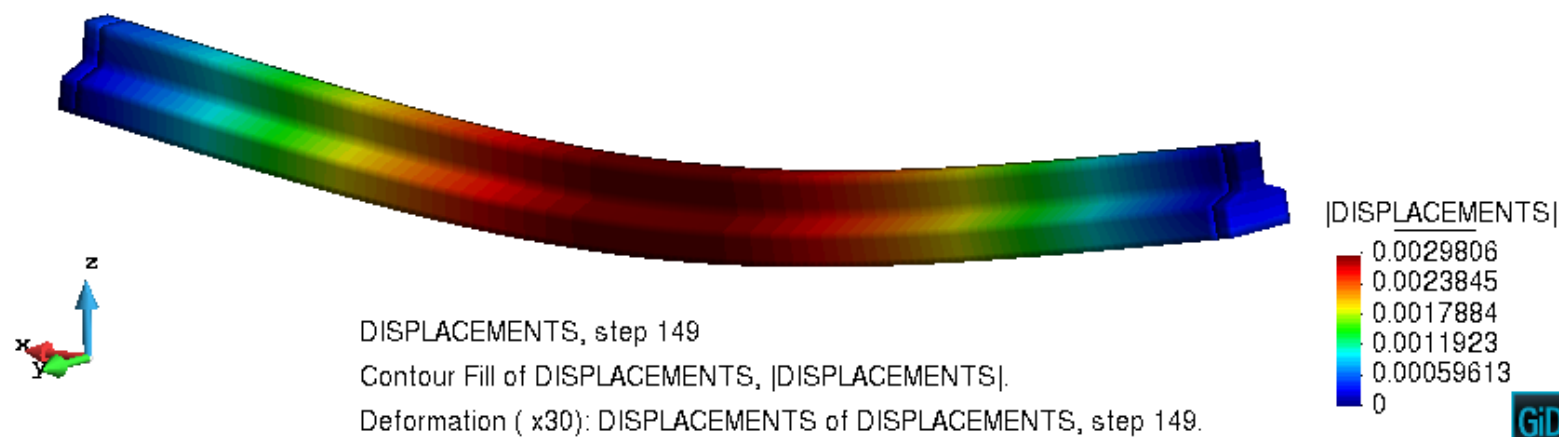

Figure 12. Displacement field at the end of the pressure load, shown on the deformed shape (x30)

After applying the pre-stressing load, a distributed load has been applied incrementally on the upper surface of the beam in order to take the structure further into the nonlinear field. The load has been applied as a monotonically increasing pressure up to a maximum level of 53500Pa (0.535bar). Figure 12 shows the displacement field on the deformed shape of the structure at the end of the pressure stage. It can be seen that the curvature of the beam has been inverted due to the applied load that generated a maximum displacement of $2.98 \mathrm{~mm}$ in the center of the structure.

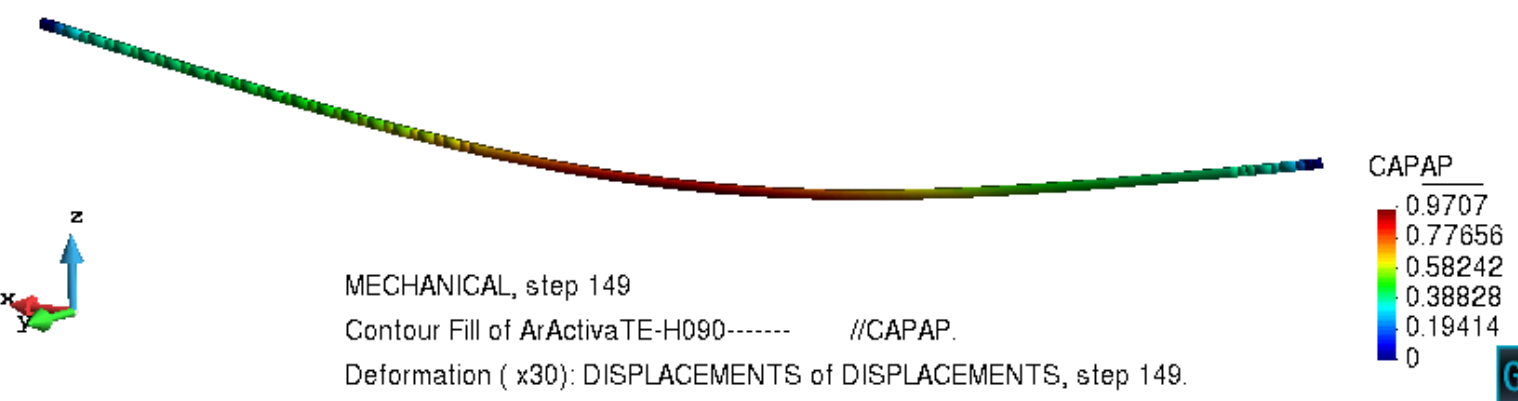

Figure 13. Normalized plastic dissipation for the steel at the end of the pressure load $\left(\mathrm{CAPAP}=\kappa^{p}\right)$

In Figure 13 the distribution of the normalized plastic dissipation parameter can be seen. As expected the elements where the steel reaches its maximum dissipation are situated in the central area of the 
beam, in accordance to the deformed shape caused by the applied loading. The most stressed elements that contain the steel tendon have dissipated $97 \%$ of the internal energy available for the steel material.

The evolution of the Von Mises equivalent stress with the equivalent strain can be seen in Figure 14 for the integration point where the maximum level of energy is dissipated. Convergence in the numerical simulation has been lost in the first increment where softening has begun because only $3 \%$ of the internal energy is available for the softening zone. This indicates that the slope of the strain-stress curve once this point has been reached is very high leading to a fragile behaviour.

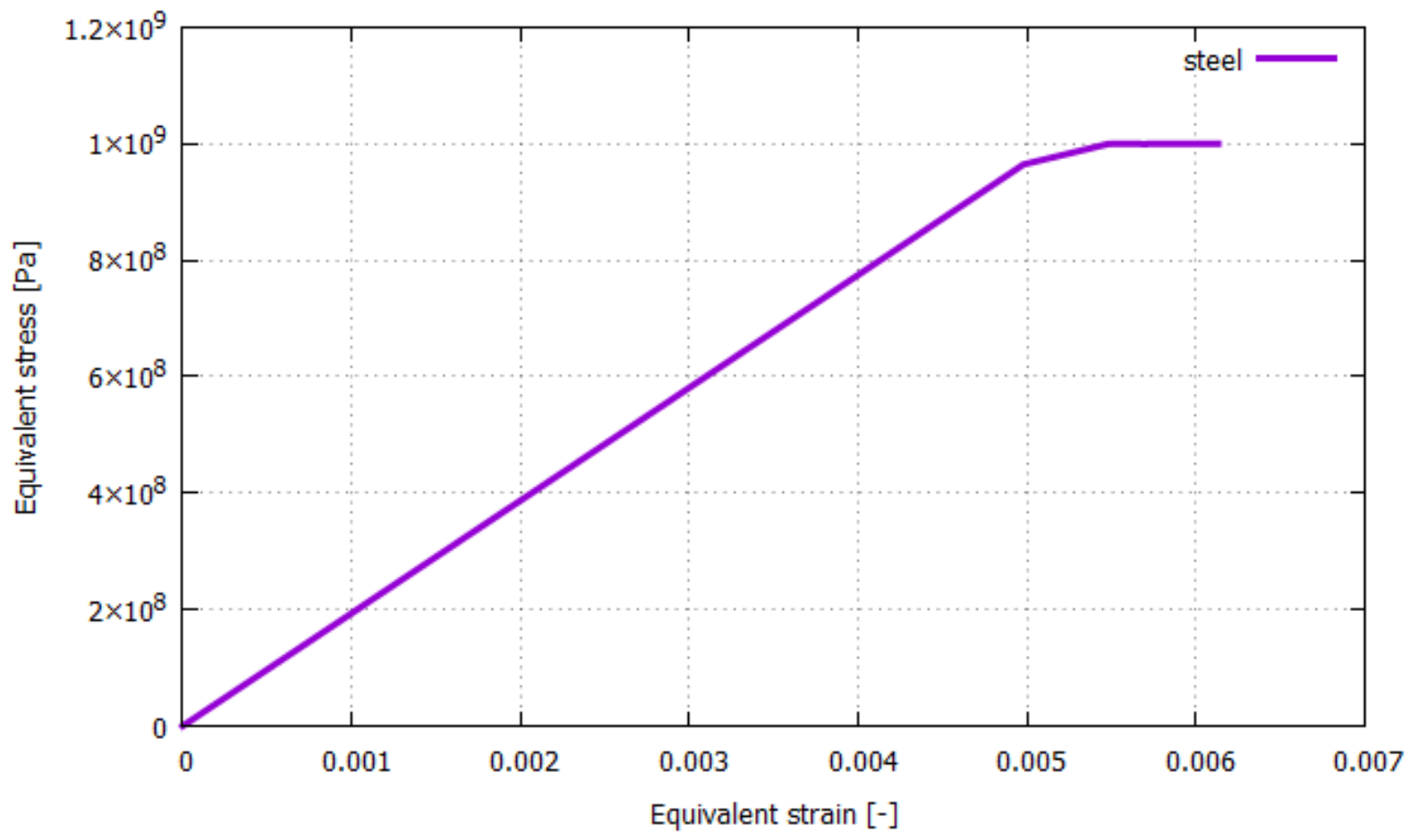

Figure 14. Stress-strain evolution for steel at the integration point that has the maximum normalized plastic dissipation

Regarding the nonlinear behaviour of concrete, Figure 15 exhibits the distribution of the damage parameter. It can be seen that degradation appears in the concrete in the traction area generated due to the flexion of the beam, as expected.

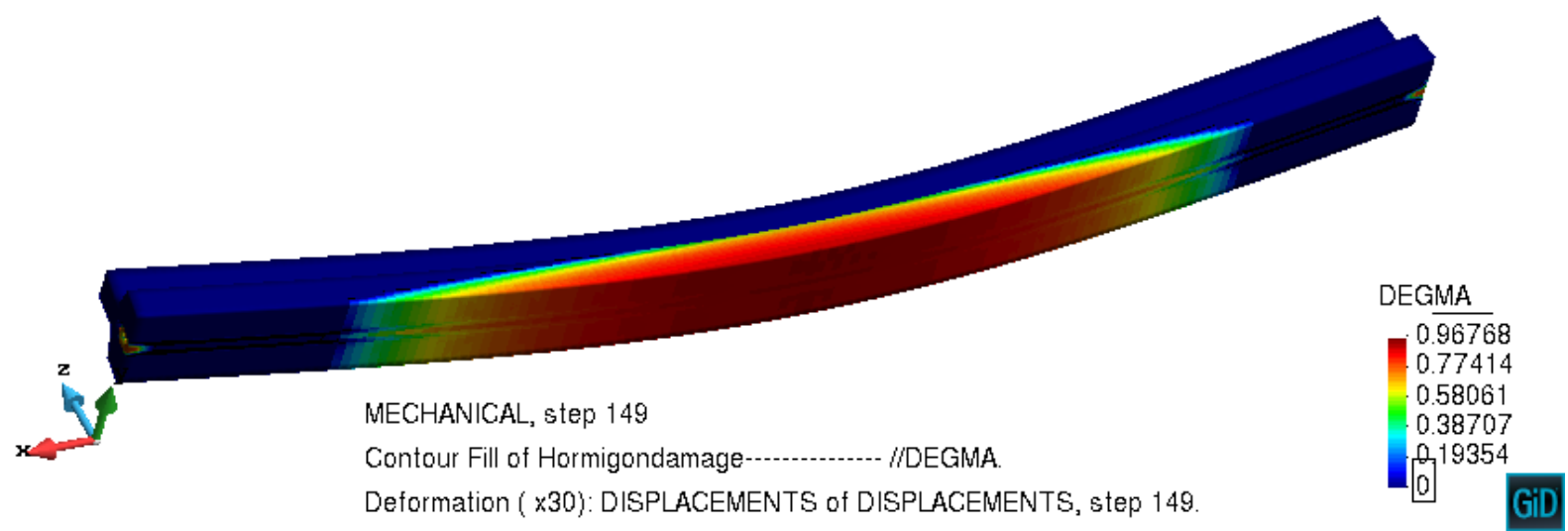

Figure 15. Damage distribution in the concrete at the end of the pressure load (DEGMA $=d$ )

In Figure 16 the equivalent stress as calculated with the Mohr-Coulomb failure criterion versus the equivalent strain can be seen. As expected, the slope of the stress-strain curve in the nonlinear domain is approximately 0 due to the very high amount of internal energy given to the concrete material. This has been done in order to ensure convergence in the numerical simulation so that the steel could be 
taken to very high dissipation levels. The main objective of the simulation is therefore to show how the proposed formulation is capable of taking into account advanced nonlinear behaviour in both components of the structure, steel and concrete.

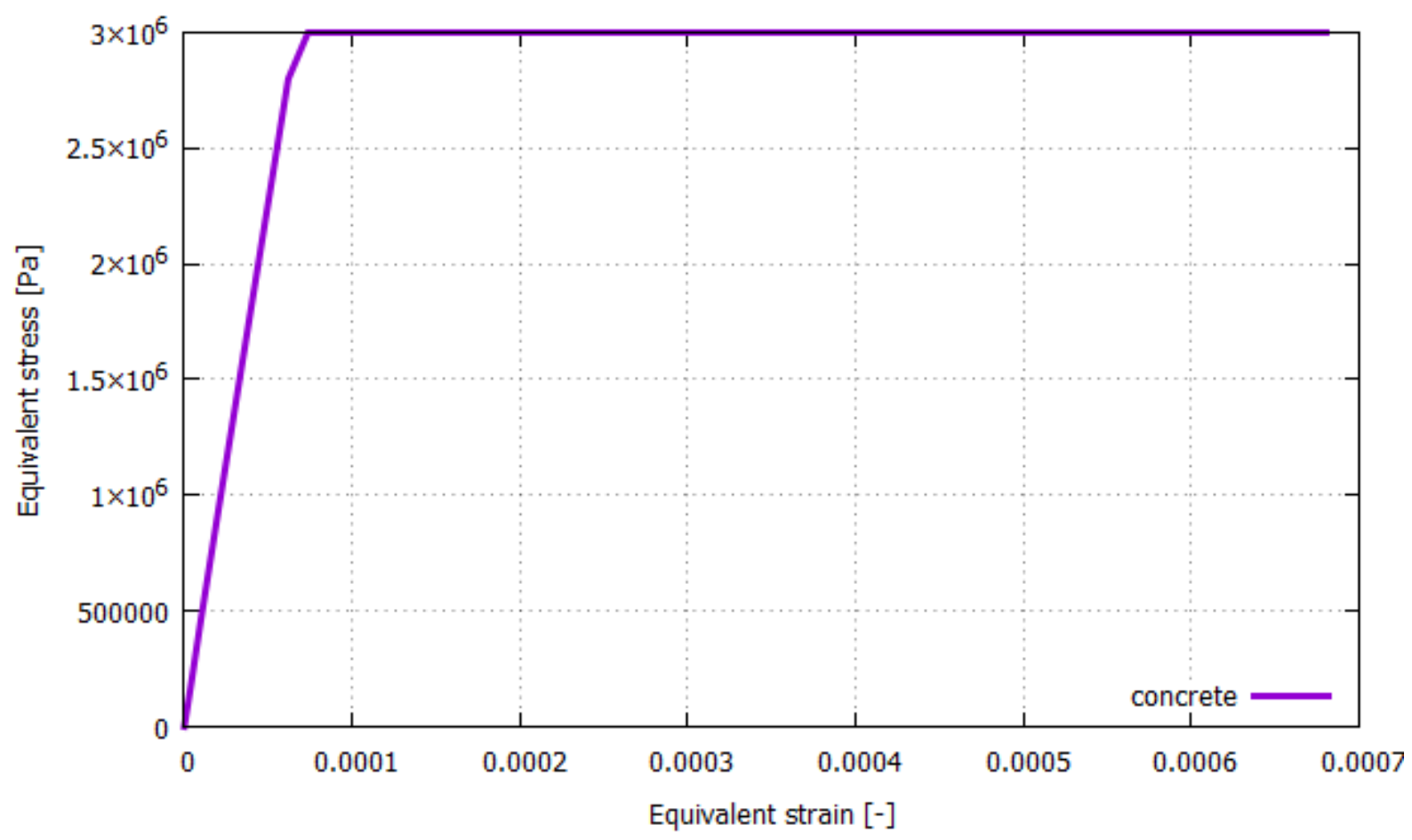

Figure 16. Stress-strain evolution for concrete at the integration point where the maximum value for the damage parameter is recorded

Figure 17 shows the distribution of the cracks in the geometry in function of the crack opening. The maximum crack opening recorded at the end of the simulation is $0.00576 \mathrm{~mm}$, showing therefore that a micro cracking process has occurred in the concrete due to the traction introduced by the applied pressure. There is loss of symmetry in the crack opening distribution, which is believed to occur due to numerical rounding in the computation of the equivalent strain.

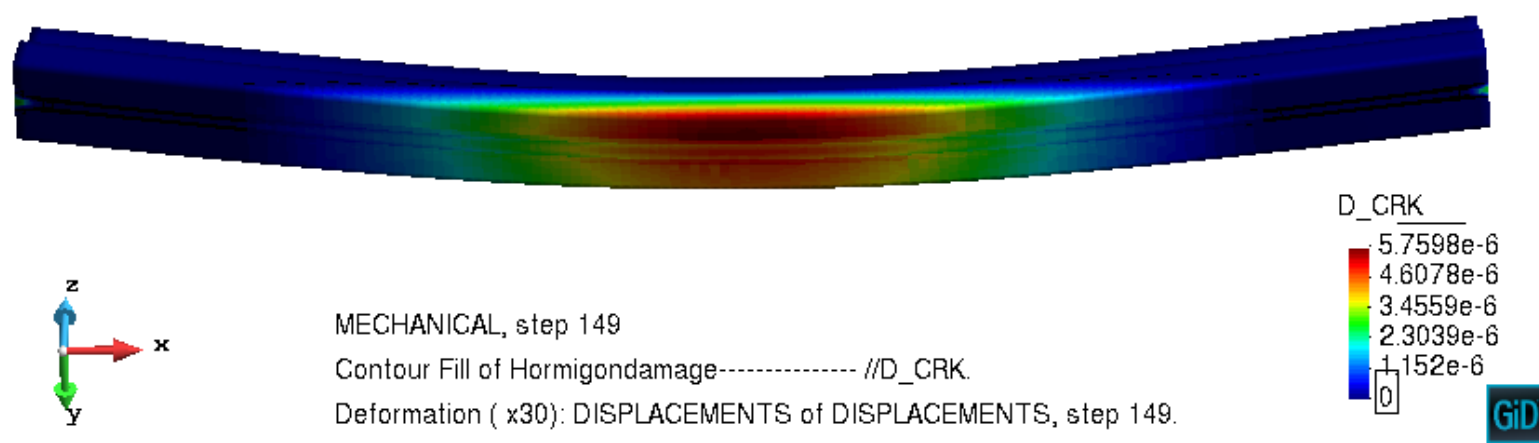

Figure 17. Crack opening distribution in the concrete at the end of the pressure load (m)

Figure 18 shows the evolution of the plasticity and damage internal variables with the applied pressure, at the integration points where their maximum value is recorded at the end of the analysis. It should be specified that the values are located in two different integration points belonging to two different elements and situated each in the area most damaged (according to Figure 15) or with the highest plastic dissipation (in accordance with Figure 13). It is interesting to see that plastic effects have already occurred in the steel prior to the application of the pressure load. Damage appears, on the 
other hand, in the area undergoing tensile stresses due to flexion only when the pressure applied exceeds approximately 26000Pa (0.26bar).

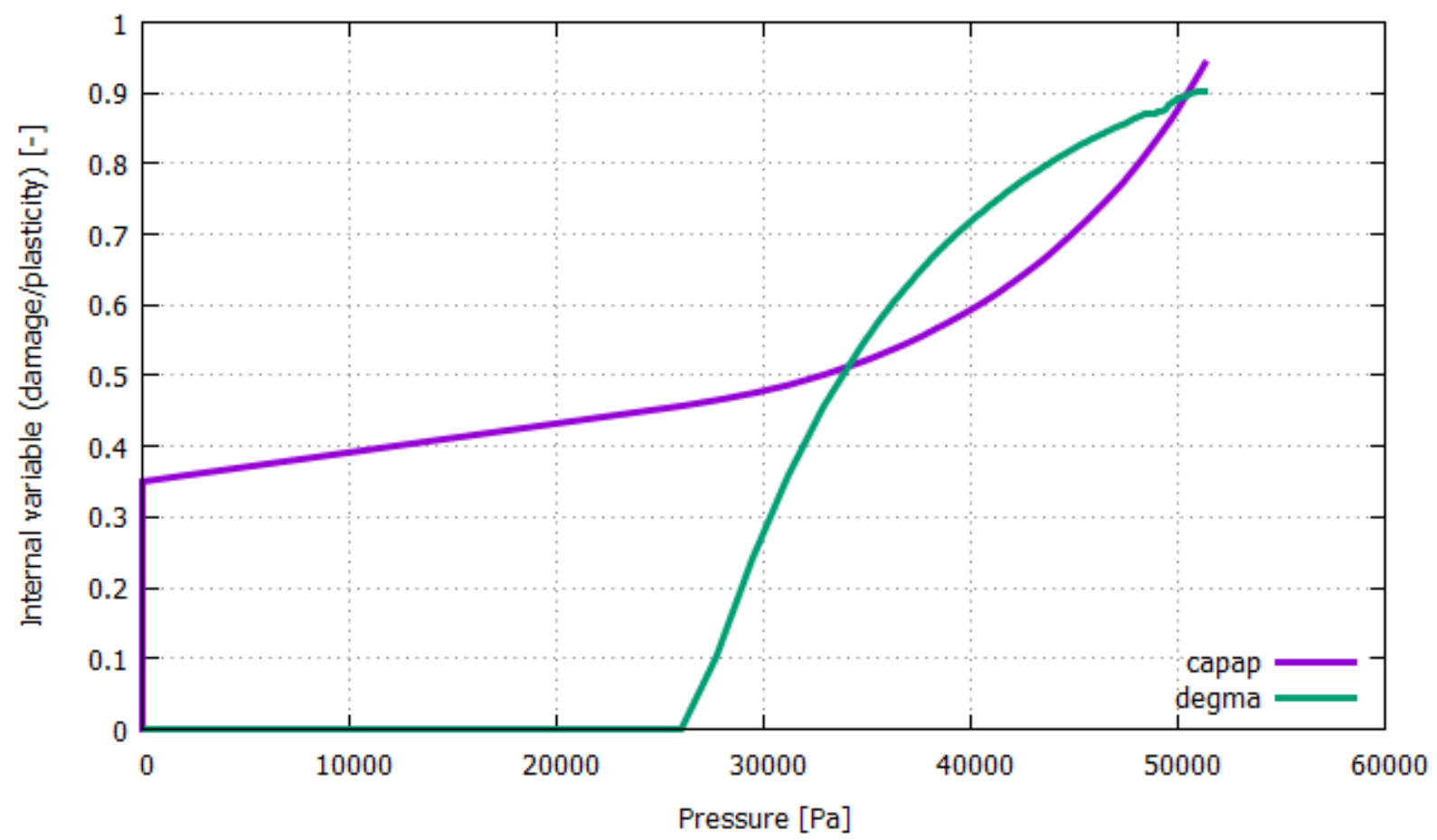

Figure 18. Evolution of the normalized plastic dissipation (CAPAP $=\kappa^{p}$ ) and of the damage variable (DEG-

$\mathrm{MA}=d$ ) with the applied pressure at the integration points where their maximum values are found

\subsection{Post-tensioned cantilever with six curvilinear steel tendons}

A bridge in a progressive cantilever assembly is analysed. This structure has a variable height and the central zone is made out of massive concrete to increase its stiffness. As it can be seen in the Fig. 19, the central zone has a width of $1.5 \mathrm{~m}$ whereas the total span of the structure is $5.75 \mathrm{~m}$ with a minimum height of $1.9 \mathrm{~m}$. Through the walls of the cantilever, there are 6 steel tendons ( 3 in each vertical wall) with a curvilinear shape to increase the post-tensing efficiency. All the steel tendons have a Young modulus equal to $195000 \mathrm{MPa}$ and have been stressed up to $1092 \mathrm{MPa}$. The concrete has a Young modulus of $40200 \mathrm{MPa}$. The exact geometry of the beam and the volumes and surfaces that define it can be seen in Figures 20 and 21.

Concrete has been modelled with the constitutive model described in Section 3, using the MohrCoulomb failure surface described in [14] and an exponential softening law. The compressive strength of concrete is $48.7 \mathrm{MPa}$ and the scaling factor between the compressive and tensile strength is 11.2, leading to a tensile strength of 4.34MPa. The tensile fracture energy considered for concrete is $400 \mathrm{MN} / \mathrm{m}$. For steel, an elastic limit of 1000MPa has been considered together with a Von Mises yield surface and a Von Mises potential criterion. The fracture energy considered is $5000 \mathrm{~N} / \mathrm{m}$ and the plastic strain at which softening is set to start is 0.001 . This parameter is necessary for the constitutive law, as explained in Section 3.

The finite element mesh shown in Figure 22 is composed by 196928 linear hexahedra and 232557 nodes. The thickness of the structure has been discretized with 4 or 5 finite elements (depending on the axis) in order to capture properly the post-tensioning effects. Regarding boundary conditions, for this model the lower surfaces of the central massive area have been completely restrained. 


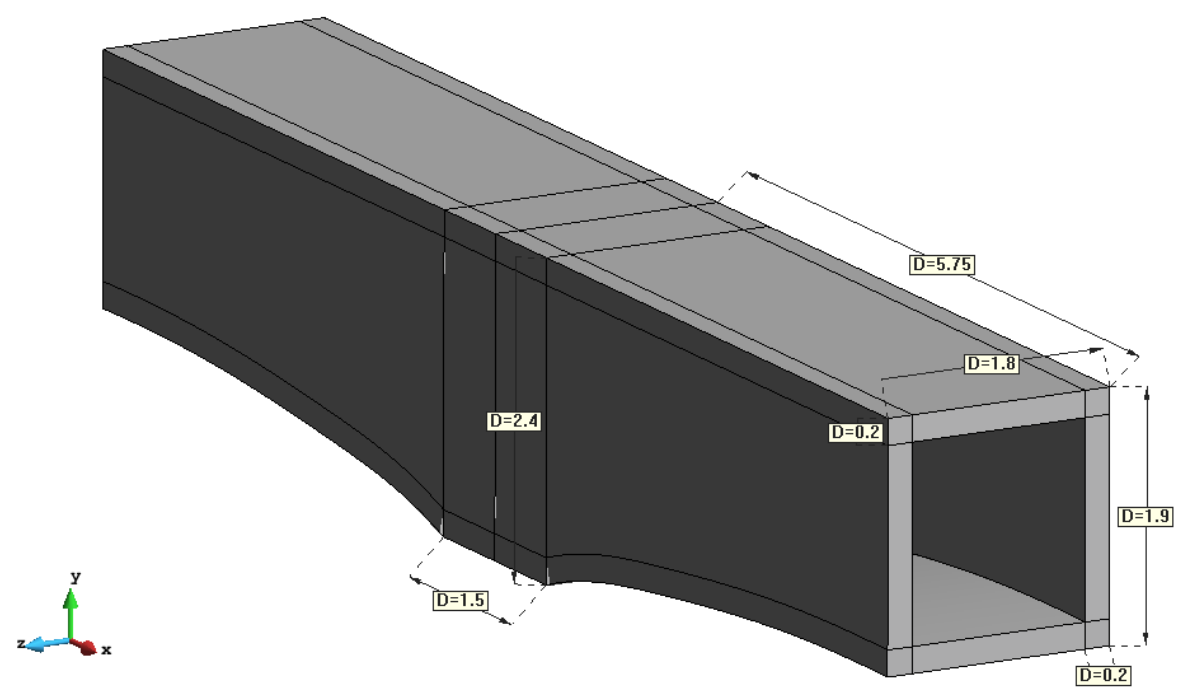

Figure 19. Geometry of the cantilever, dimensions in $\mathrm{m}$.

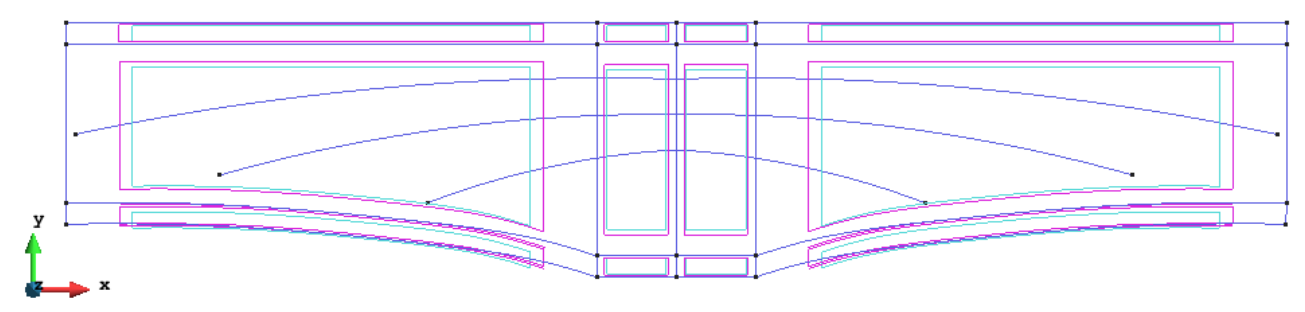

Figure 20. Geometry of the cantilever and the steel tendons

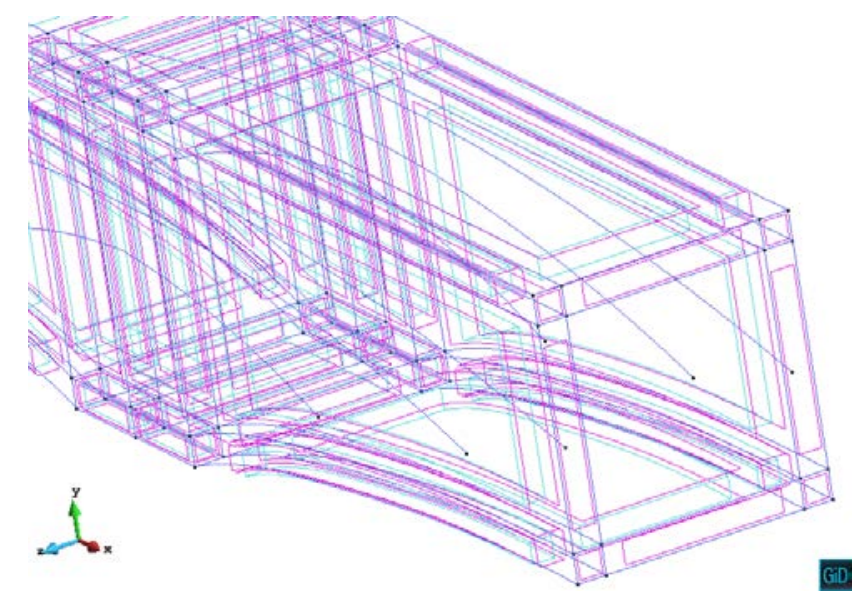

Figure 21. Perspective of the cantilever and the steel tendons

First, the self weight load has been applied. The strain imposed was 0.0056 for the active steel contained in those finite elements that were intersected by the steel tendons. Afterwards, a pressure of 2.90bars (290000Pa) has been applied incrementally until both steel and concrete reached a level of plastic dissipation or damage of at least $90 \%$ at at least one integration point. 


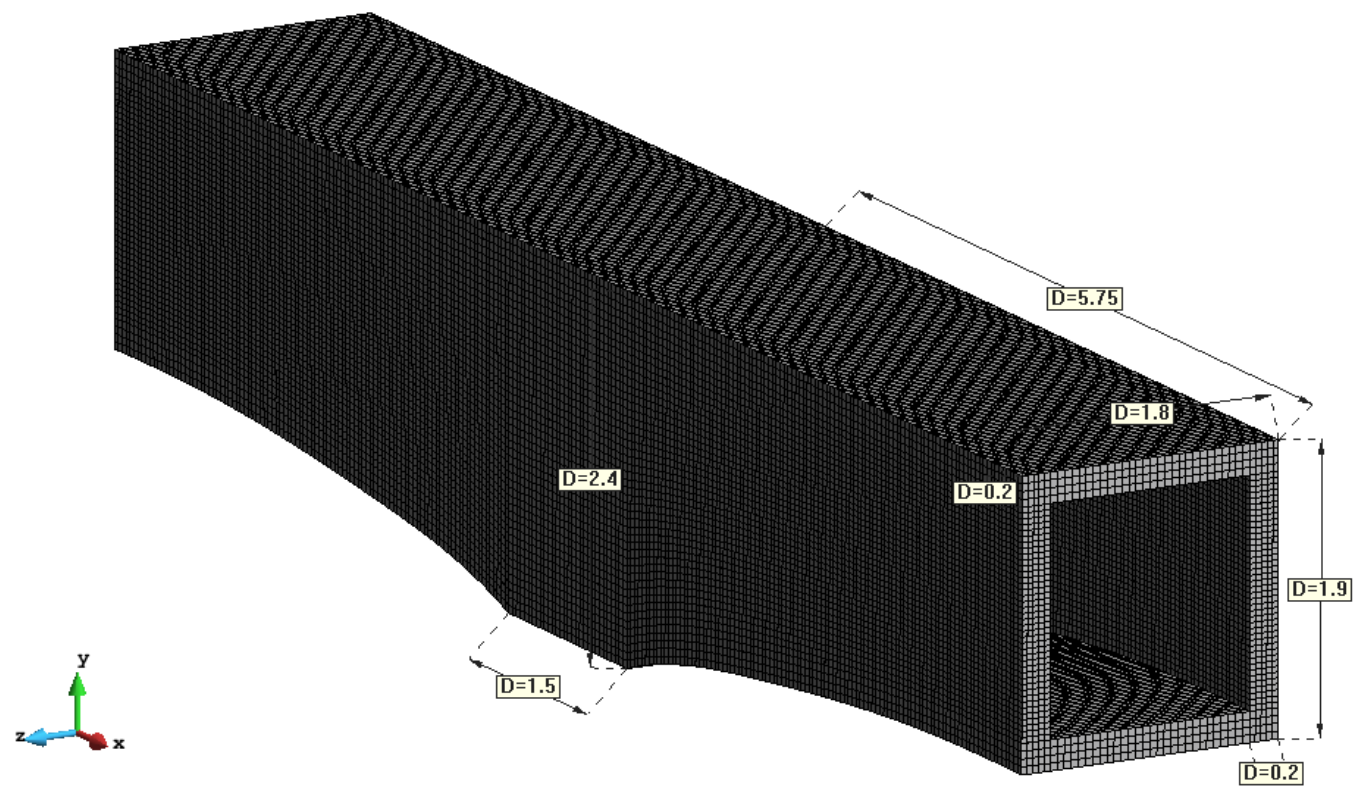

Figure 22. Perspective of the finite element mesh (196,928 finite elements)

After intersecting the lines that represent the steel tendons with the finite element mesh, we obtain a finite element discretization of the steel tendons inside the global mesh. The final result implies that there are some finite elements (the ones intersected by the active steel) with a certain volumetric participation and direction of post-tensioning whereas the rest behaves as a homogeneous concrete. The intersected finite elements can be seen in the figures 23 and 24. Each colour symbolizes a different volumetric participation and/or different orientation of the local longitudinal direction. In this case, 551 composite materials where generated due to different orientation and percentage of the two main composing materials, steel and concrete.

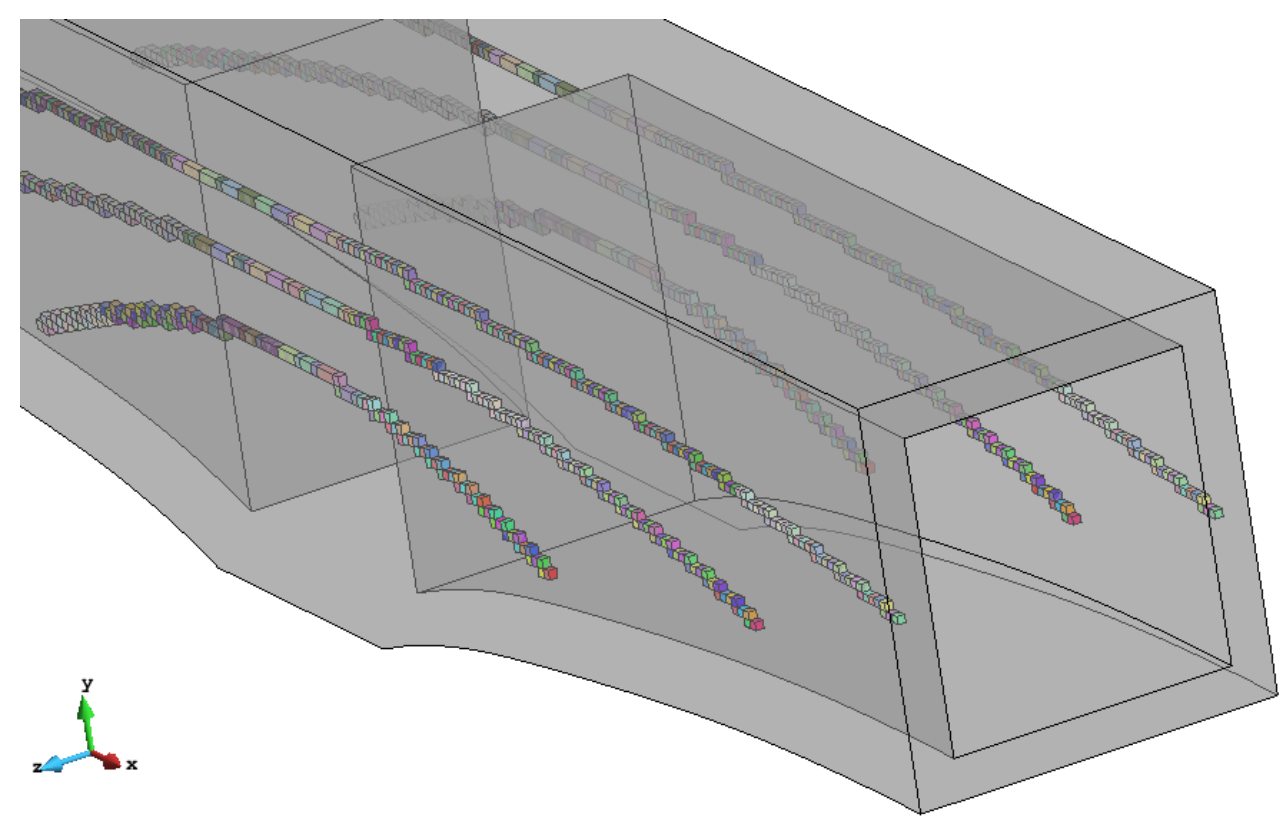

Figure 23. Perspective of the finite elements intersected by the active steel 


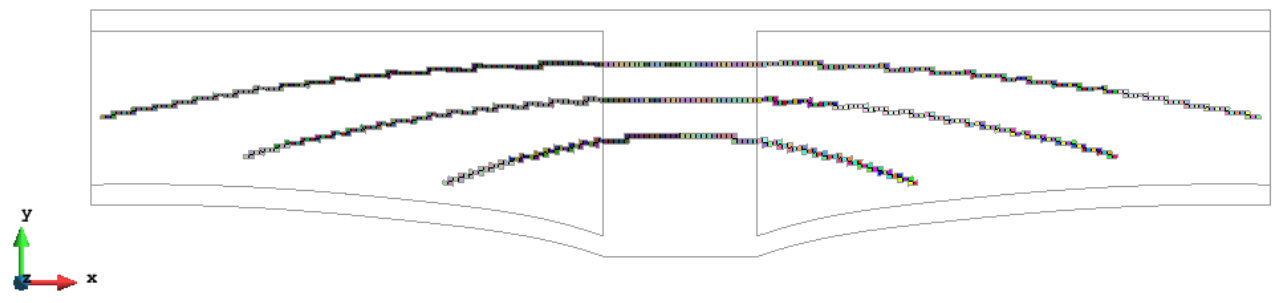

Figure 24. x-y perspective of the finite elements intersected by the active steel

The results of the computation of the structure taking into account the effect of the self-weight are shown below. In Figure 25 the displacement field as resulting from the self-weight load can be seen, while in the figures 26 to 28 the structure is described after the post-tensioning load has been applied. The maximum displacement caused by the self weight is $0.1438 \mathrm{~mm}$, recorded at the ends of the cantilever, as expected. It can be seen by comparing Figures 25 and 26 that the post-tensing of the steel tendons reduces the maximum displacement in the geometry up to a level of $0.1074 \mathrm{~mm}$ and therefore has a favourable compensating effect with respect to the self-weight loading.

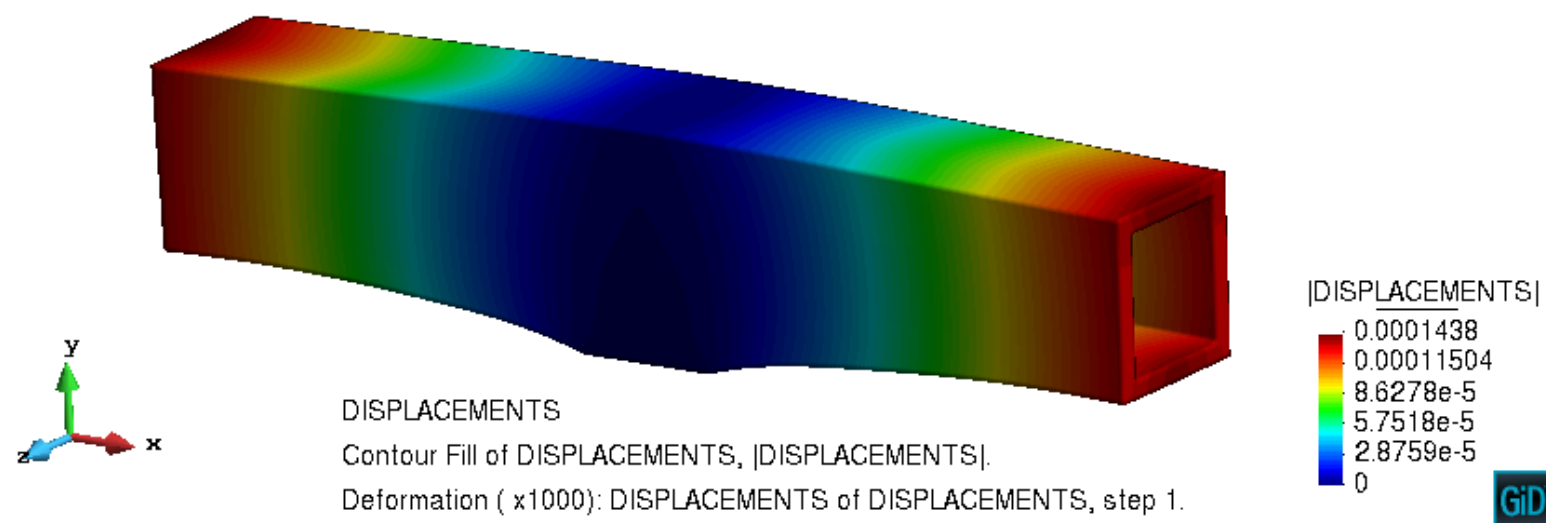

Figure 25. Displacement field after self-weight, shown on the deformed shape (x1000)
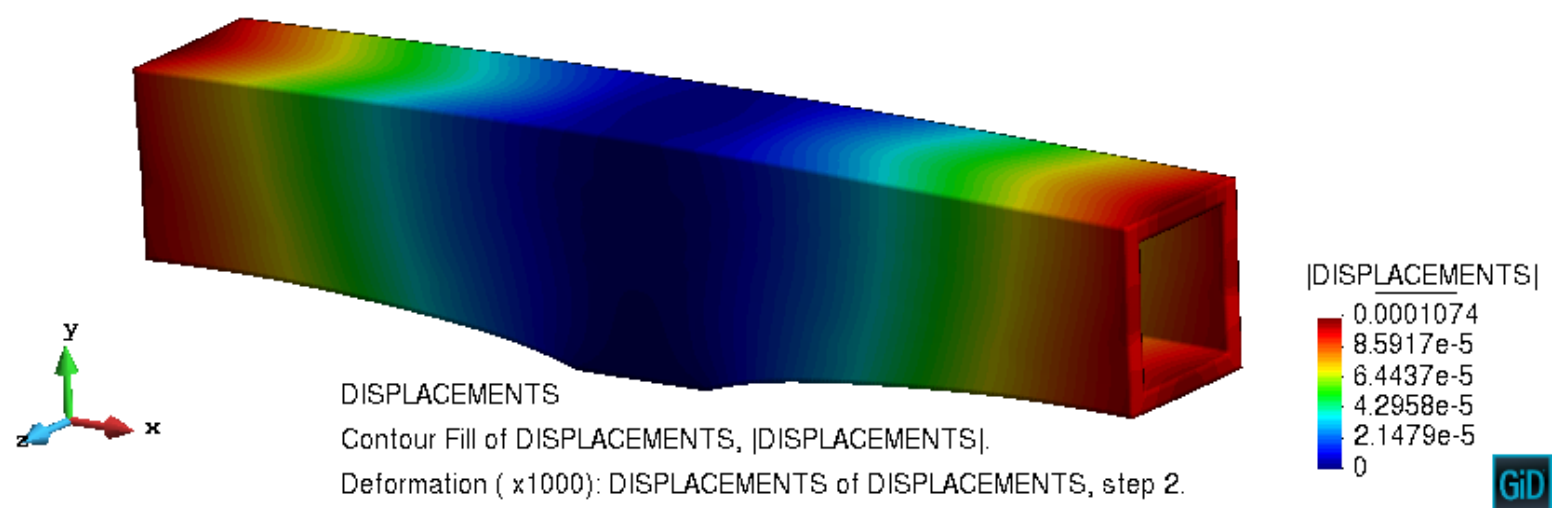

Figure 26. Displacement field at the end of the post-tensioning load, shown on the deformed shape (x1000)

Figures 27 and 28 show the distribution of the plasticity and damage internal variables in the steel tendons and concrete, respectively. Both results are shown on the deformed shape of the cantilever with a scaling factor of 1000 . It can be seen that at the end of the post-tensioning stage the steel has dissipated at its most tensioned point $53.6 \%$ of its internal energy. Concrete, on the other hand, exhibits very localized degradation only in those elements that are closest to the post-tensioned steel tendons, without any visible degradation at a structural level, as can be seen in Figure 28. 


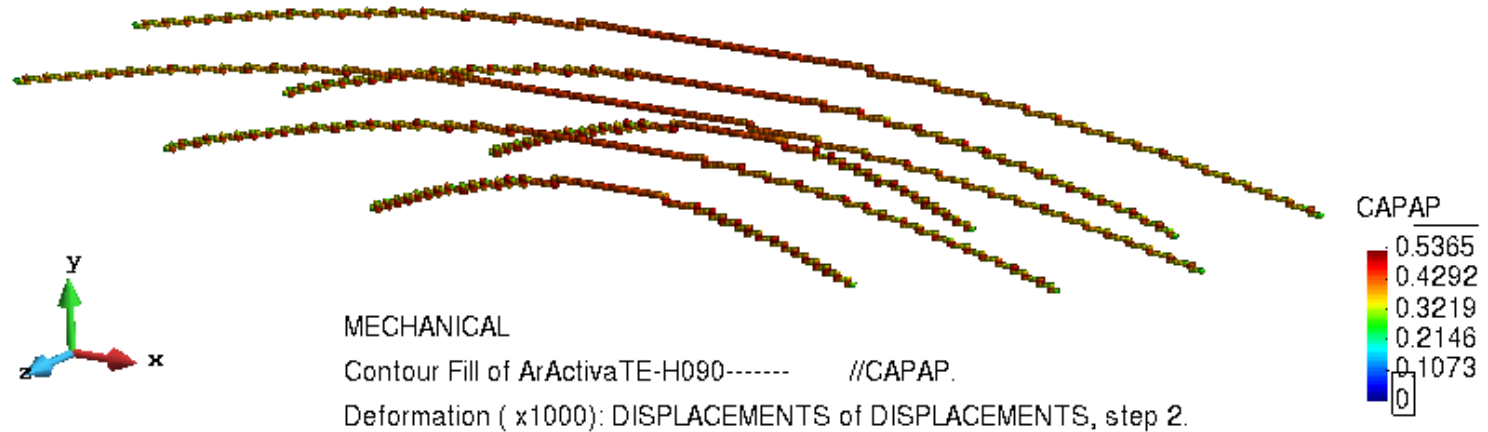

Figure 27. Normalized plastic dissipation for the steel at the end of the post-tensioning load (CAPAP $=\kappa^{p}$ )

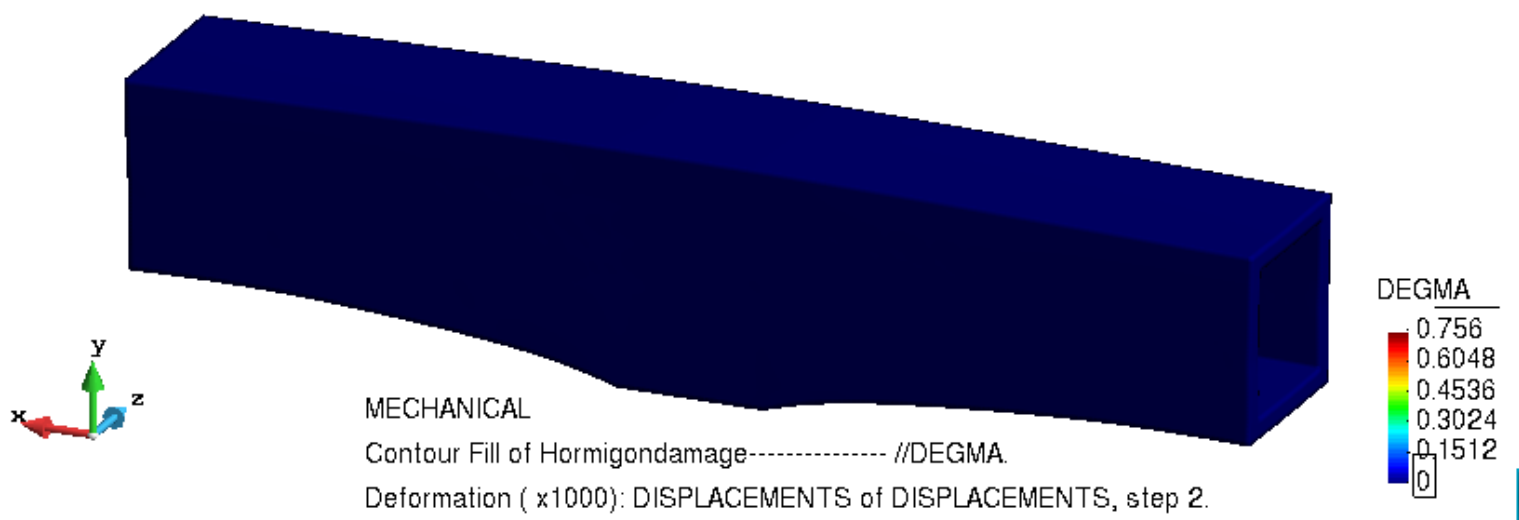

Figure 28. Damage distribution in the concrete at the end of the post-tensioning load (DEGMA $=d$ )

After the steel tendons have been post-tensioned a distributed load has been applied as a pressure on one of the sides of the cantilever incrementally until reaching a value of $290000 \mathrm{~Pa}$ (2.9bars). Results at the end of the analysis are shown in Figures 29 to 37. Figure 29 exhibits the displacement field on the deformed shape of the geometry, with a scaling factor of 200. It can be seen how the symmetry has been lost due to the load applied asymmetrically and that the maximum displacement recorded is now $6.65 \mathrm{~mm}$, significantly higher, as expected.

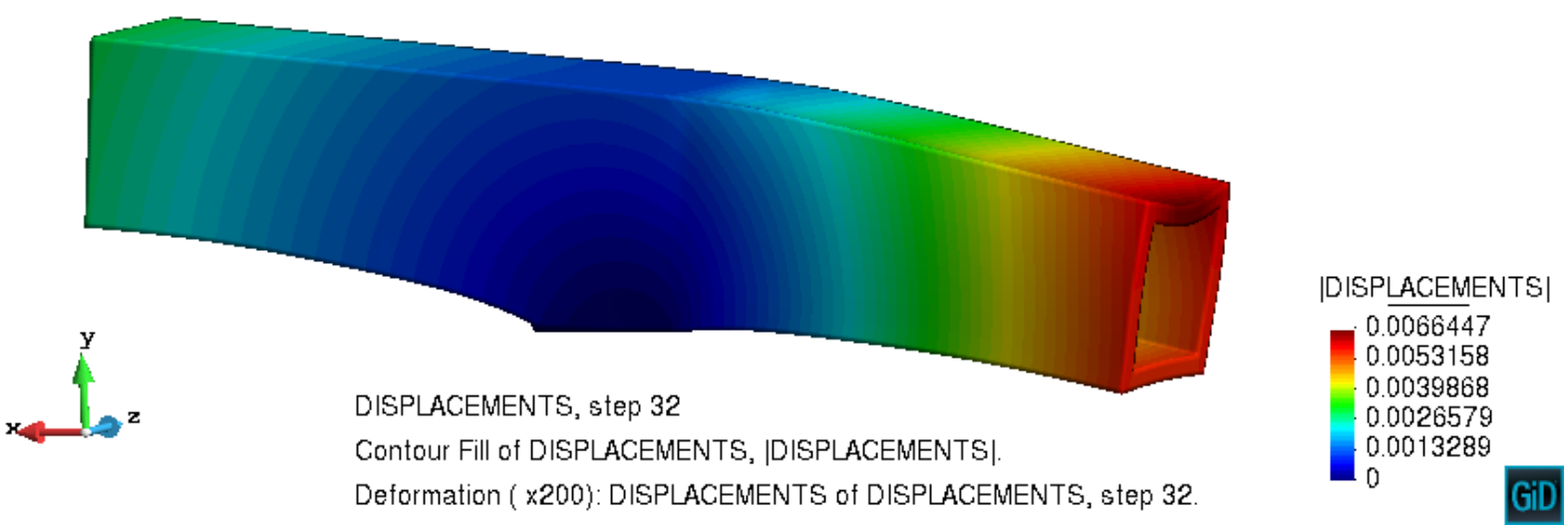

Figure 29. Displacement field at the end of the pressure load, shown on the deformed shape (x200)

Another view of the displacement field is given in Figure 30 so the effect of the distributed load can be better observed on the deformed shape of the geometry, again with a scaling factor of 200 . 


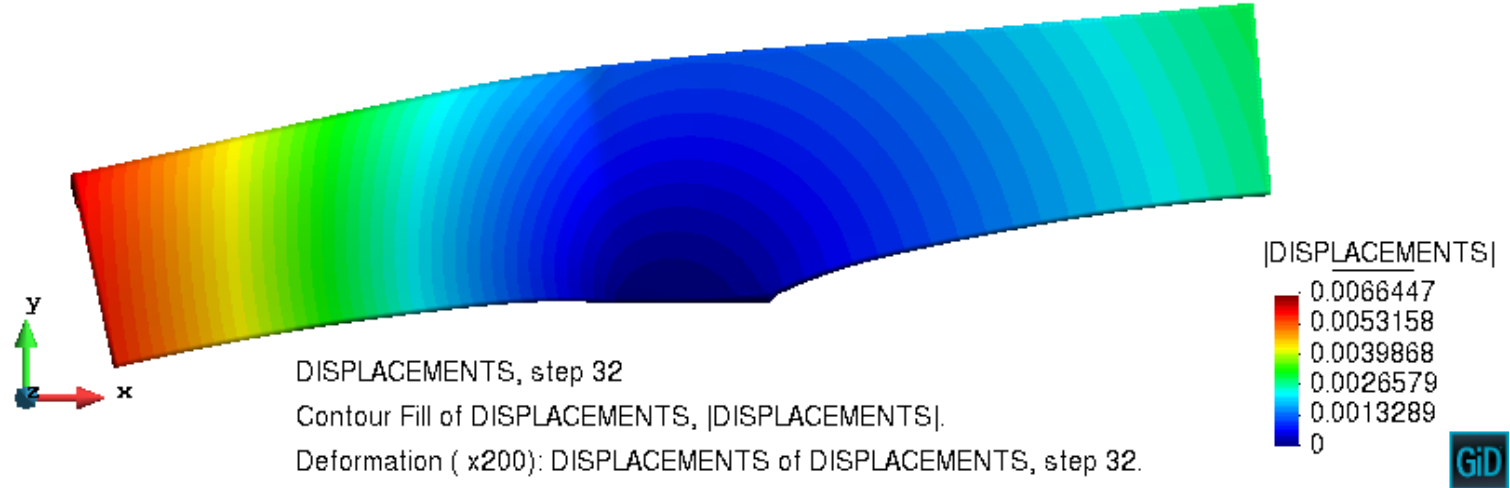

Figure 30. Displacement field at the end of the pressure load, shown on the deformed shape (x200), view of the

$$
\text { XOY plane }
$$

In Figure 31 the distribution of the normalized plastic dissipation parameter can be seen. As expected the elements where the steel reaches its maximum dissipation are situated in the central area of the cantilever towards the side where the pressure is applied, in accordance to the deformed shape caused by the applied loading. The most stressed elements that contain the steel tendon have dissipated $98 \%$ of the internal energy available for the steel material. The steel tendons where the maximum dissipation is recorded are the uppermost ones situated in the area subjected to tensile stresses.
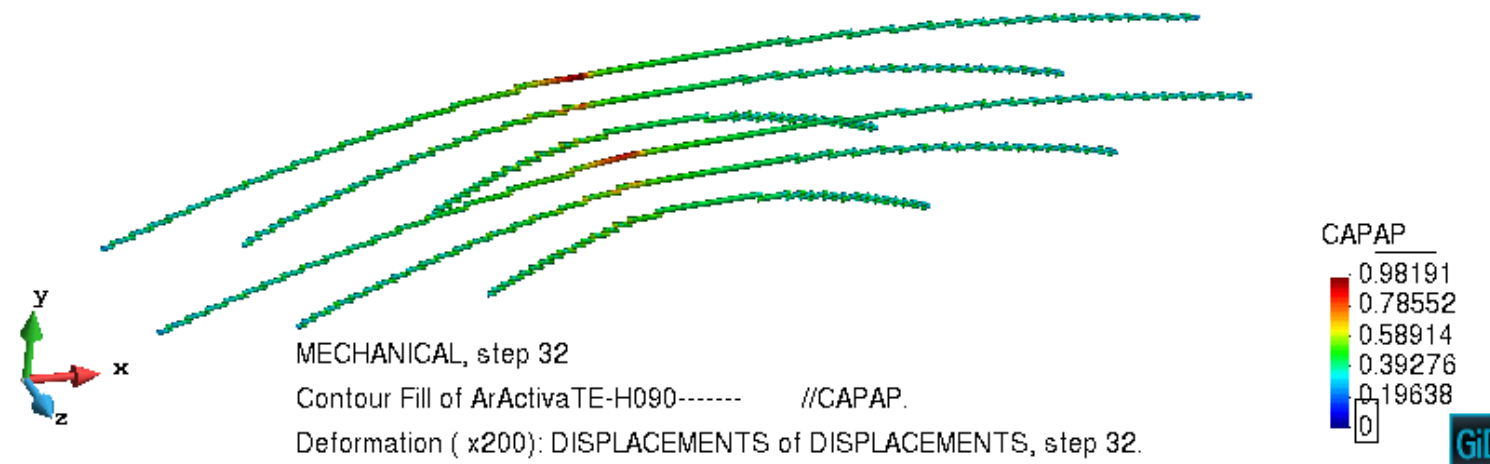

Figure 31. Normalized plastic dissipation for the steel tendons at the end of the pressure load (CAPAP $=\kappa^{p}$ )

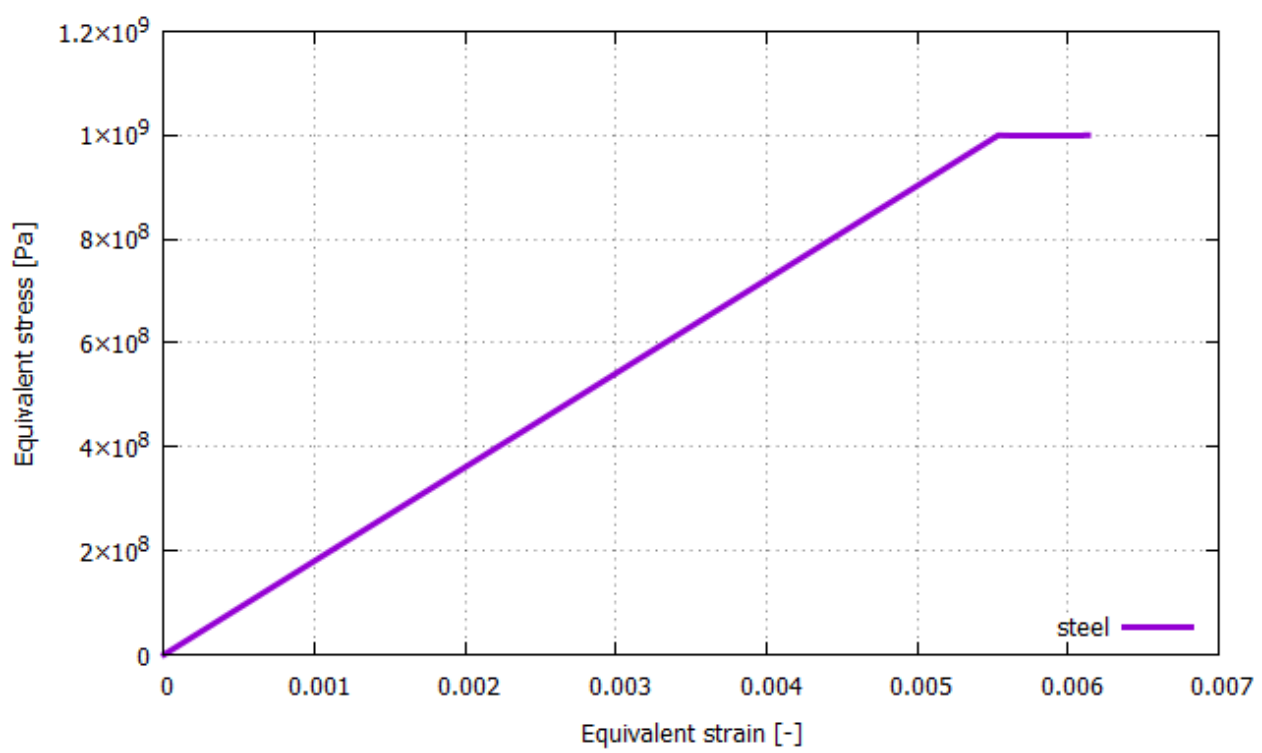

Figure 32. Stress-strain evolution for steel at the integration point that has the maximum normalized plastic dissipation 
The evolution of the Von Mises equivalent stress with the equivalent strain can be seen in Figure 32 for the integration point where the maximum level of energy is dissipated. Convergence in the numerical simulation has been lost in the first increment where softening has begun because less than $2 \%$ of the internal energy is available for the softening zone. This indicates that the slope of the strain-stress curve once this point has been reached is very high leading to a fragile behaviour.
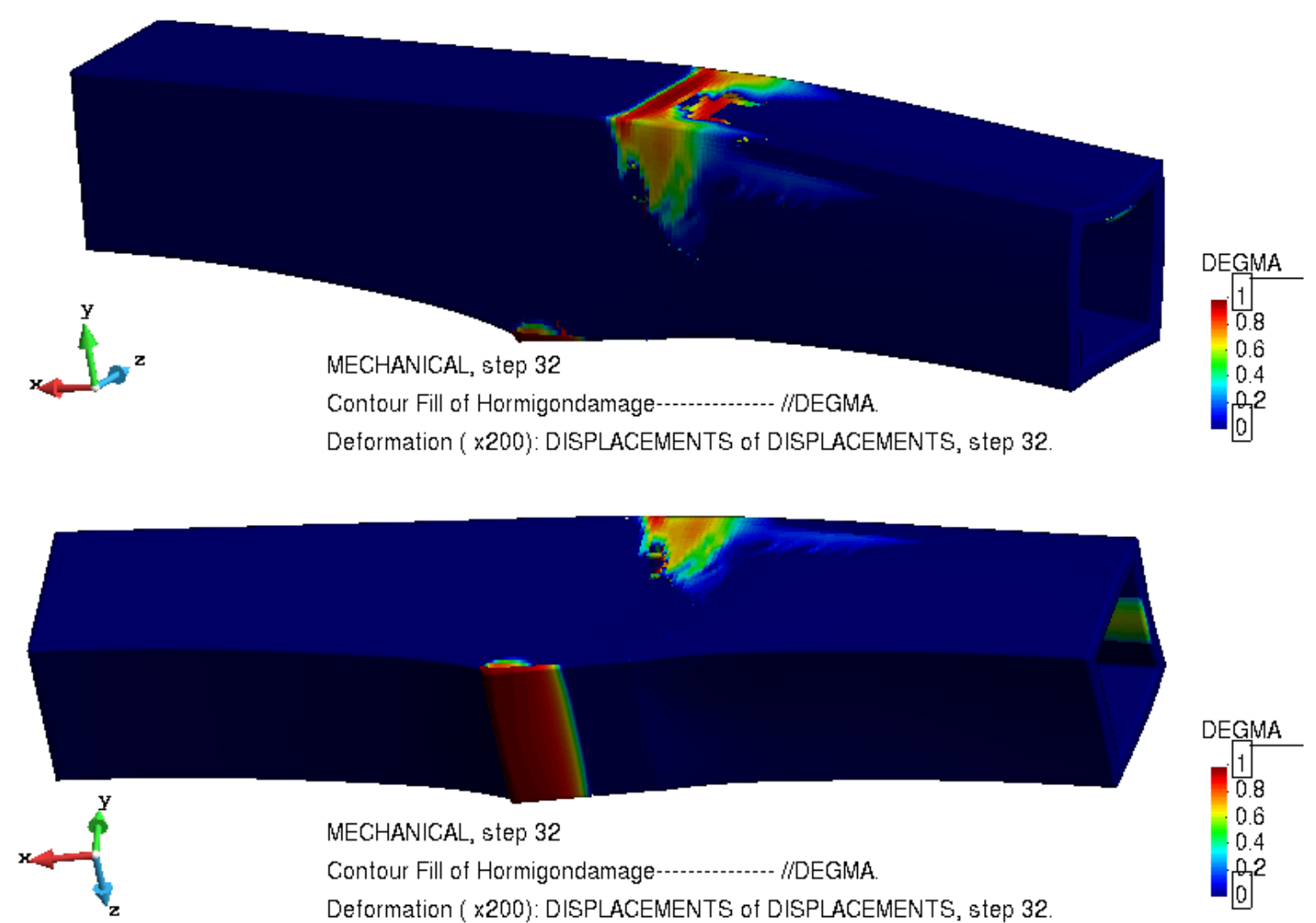

Figure 33. Damage distribution in the concrete at the end of the pressure load $($ DEGMA $=d$ )

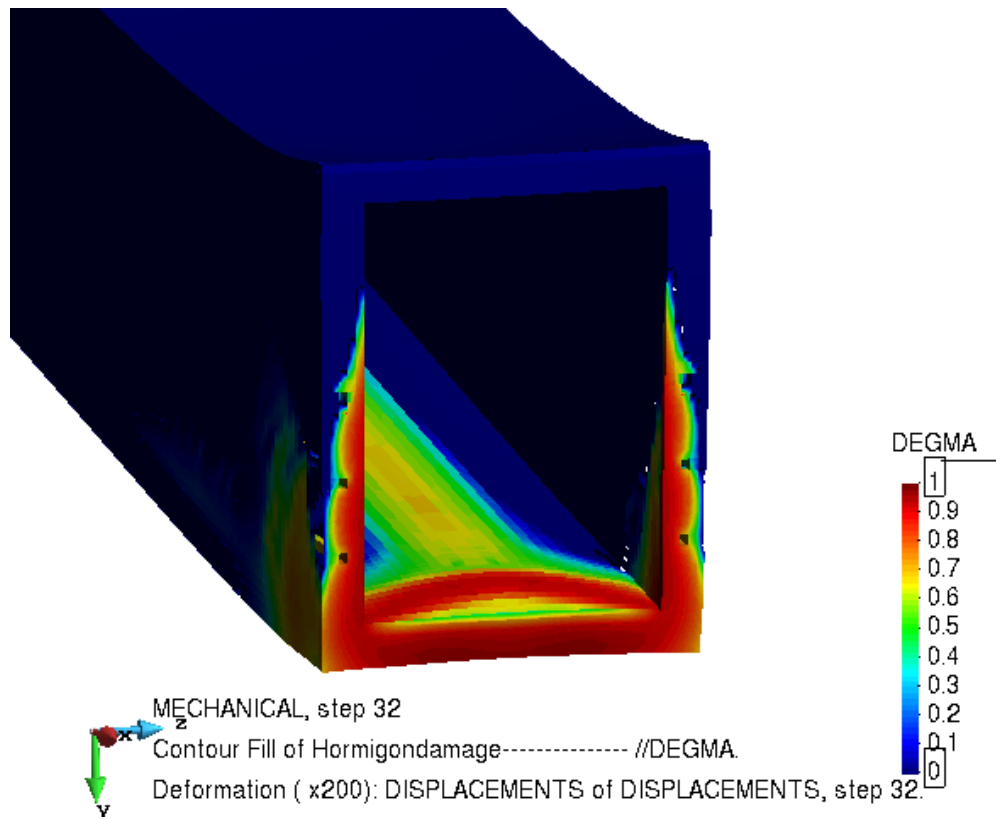

Figure 34. Damage distribution in the concrete at the end of the pressure load, view on the sectioned geometry

$$
(\mathrm{DEGMA}=d)
$$


Figure 33 shows two different views of the damage variable distribution on the deformed geometry. It can be seen that damage concentrates in three main areas: traction area in the supports of the cantilever, traction area in the upper part of the geometry where the loaded flange of the cantilever connects with the rigid concrete center and in the upper inner part of the closed section. All of these areas are subjected to tensile stresses.

In Figure 34 we can see the distribution of the damage on the sectioned geometry so that the damage distribution throughout the thickness of the concrete wall can be better seen.

In Figure 35 the equivalent stress as calculated with the Mohr-Coulomb failure criterion versus the equivalent strain can be seen. As expected, the slope of the stress-strain curve in the nonlinear domain is approximately 0 due to the very high amount of internal energy given to the concrete material. This has been done in order to ensure convergence in the numerical simulation so that the steel could be taken to very high dissipation levels. The main objective of the simulation is therefore to show how the proposed formulation allows advanced nonlinear behaviour in both components of the structure, steel and concrete.

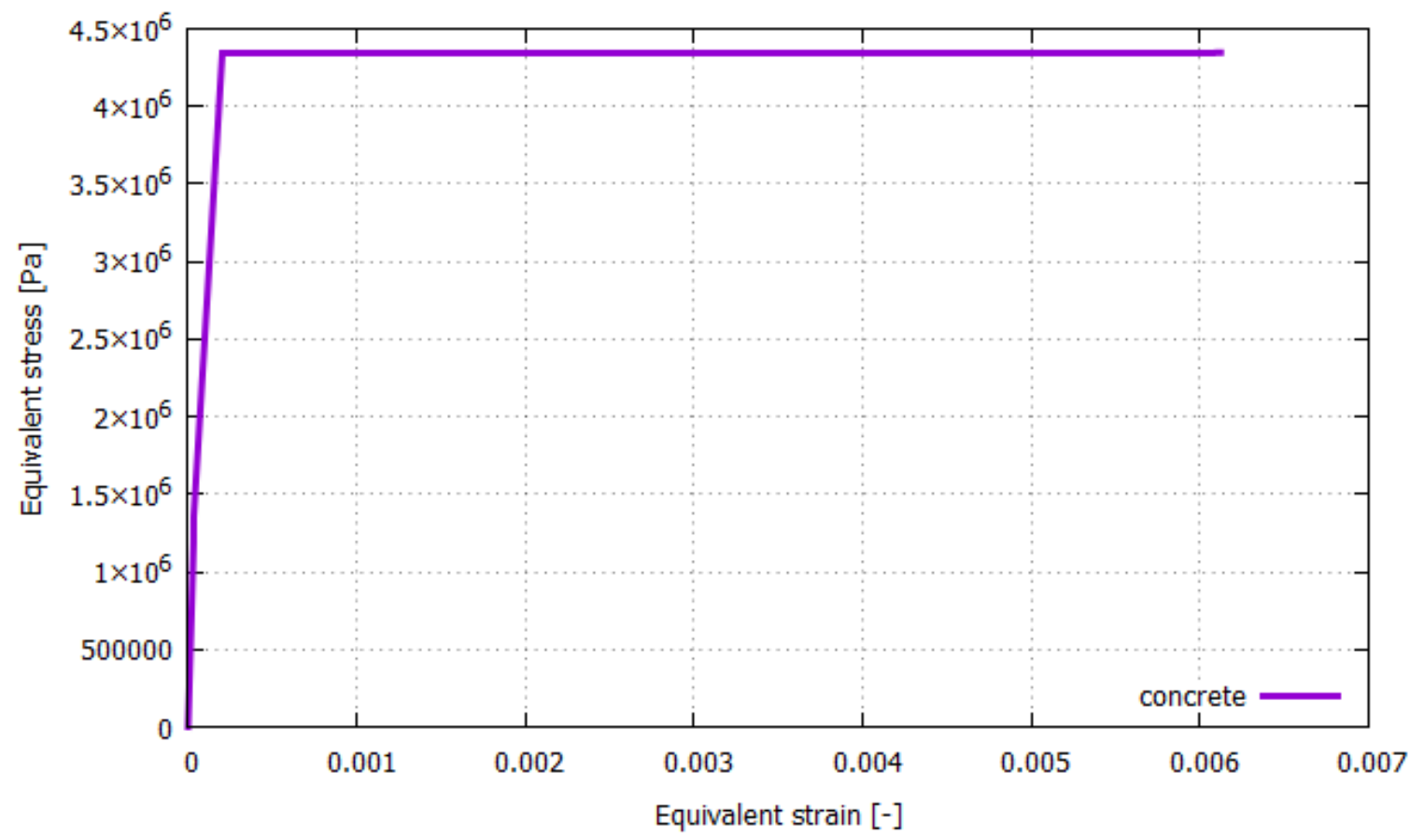

Figure 35. Stress-strain evolution for concrete at the integration point where the maximum value for the damage parameter is recorded

Figure 36 shows the distribution of the cracks in the geometry in function of the crack opening. The maximum crack opening recorded at the end of the simulation is $0.31 \mathrm{~mm}$, showing therefore that the cracking process is starting to be visible at the macroscale level. The largest crack opening is found at the constraints due to the rotation introduced by the applied pressure. Another clearly marked crack appears at the connection between the center area and the loaded flange where the change in stiffness occurs between the massive concrete center and the closed section of the wing.

Figure 37 shows the evolution of the plasticity and damage internal variables with the applied pressure, at the integration points where their maximum value is recorded at the end of the analysis. Again, it should be specified that the values are located in two different integration points belonging to two different elements and situated each in the area most damaged (according to Figure 33) or with the highest plastic dissipation (in accordance with Figure 31). For this cantilever beam, we also have plas- 
tic effects already in the steel prior to the application of the pressure load. As soon as the pressure is applied damage appears at the constraints.

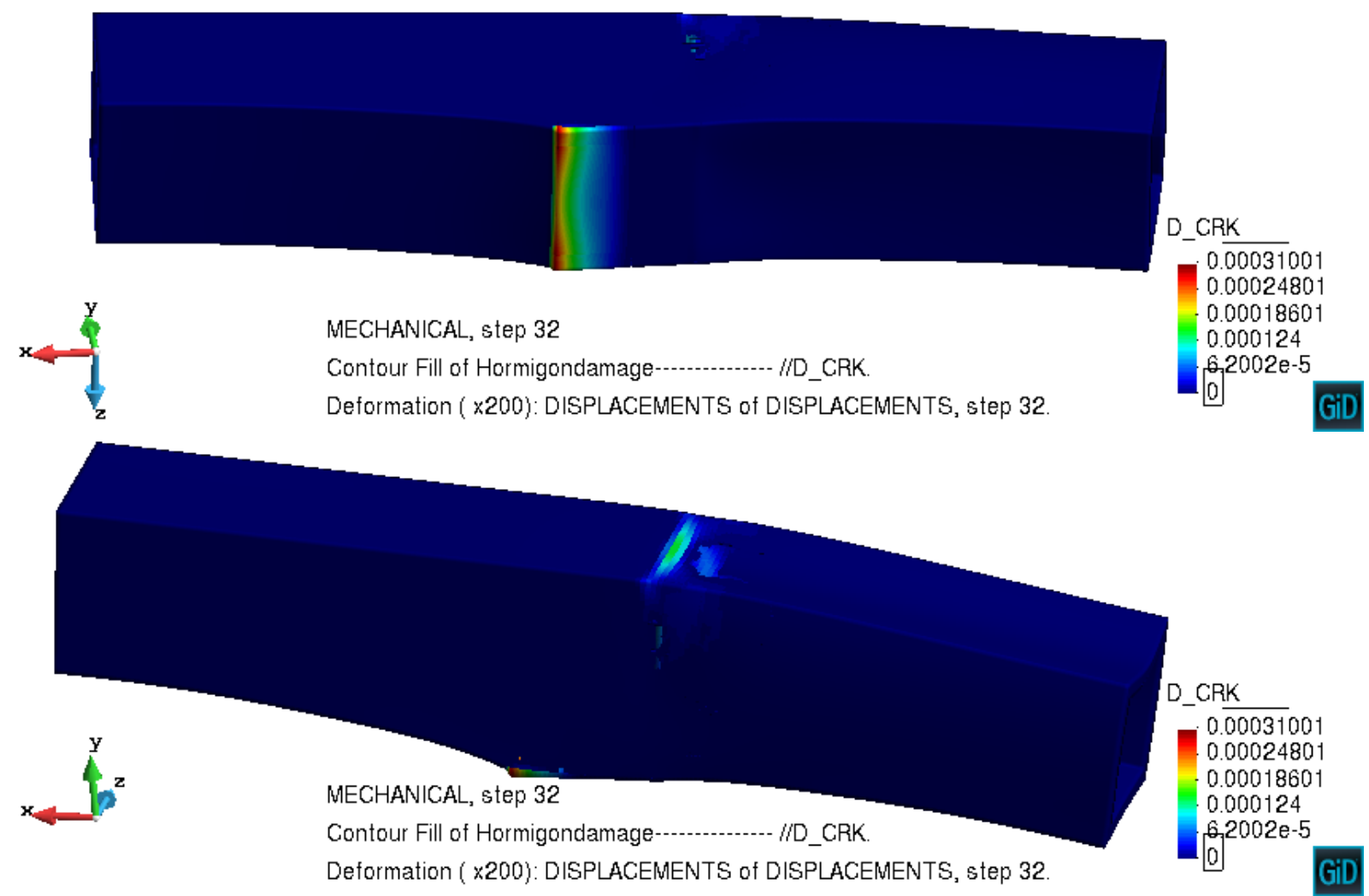

Figure 36. Crack opening distribution in the concrete at the end of the pressure load (m), different views

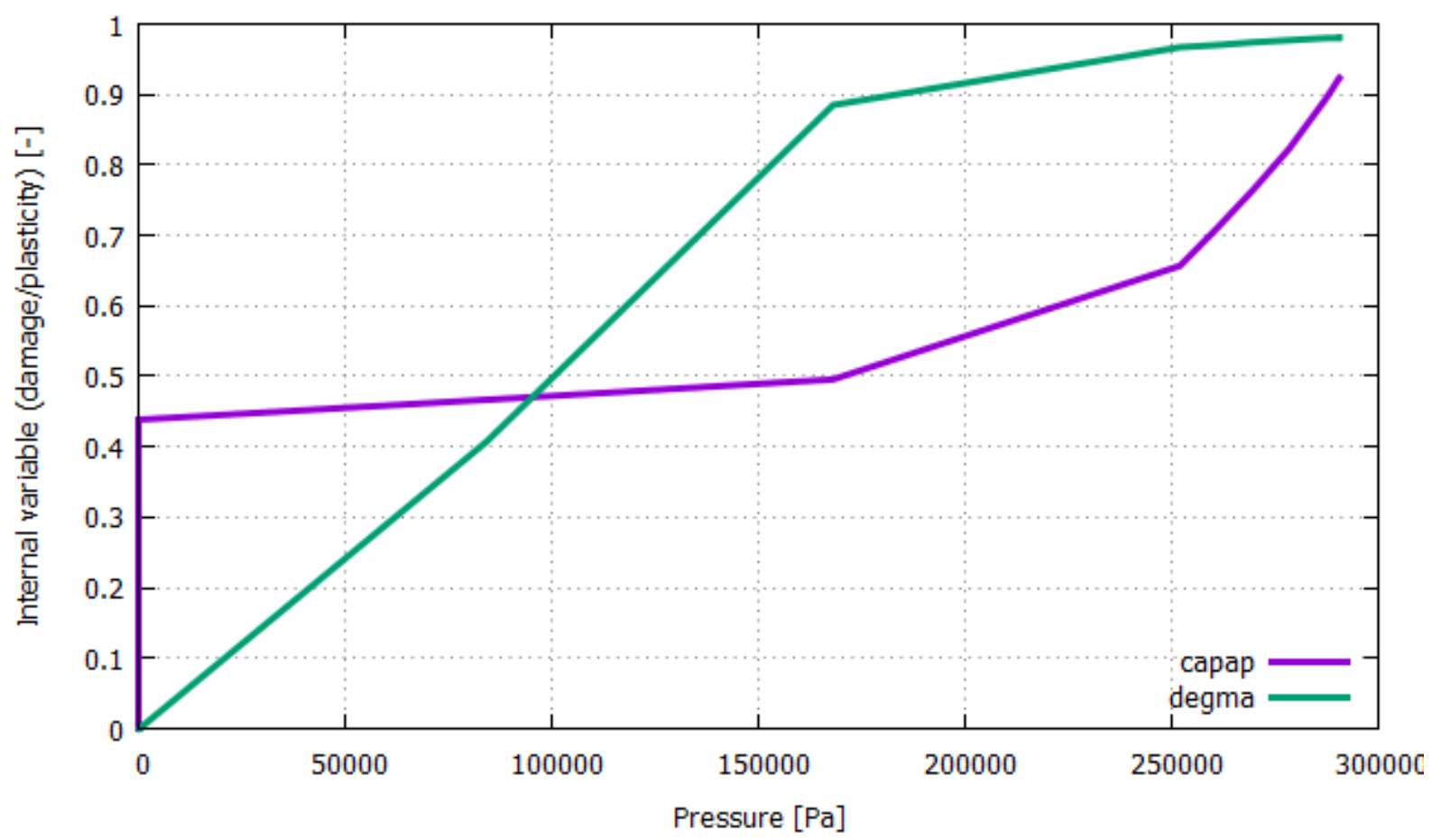

Figure 37. Evolution of the normalized plastic dissipation (CAPAP $=\kappa^{p}$ ) and of the damage variable (DEG$\mathrm{MA}=d$ ) with the applied pressure at the integration points where their maximum values are found 


\section{Conclusions}

In this paper, the innovative methodology for the numerical analysis of pre-stressed and post-tensioned structures developed previously has been applied in the nonlinear analysis of civil engineering structures. This method is able to account for the transmission of structural effects from one material to the other implicitly and allows the monitoring of the internal stress-strain state on each component material of the volume afferent to each integration point. Furthermore, its generic integration and equilibrium scheme allows different type of constitutive behaviour for each material that is present in the structure. The methodology thus is highly versatile in this respect.

At the same time, its computational simplicity deems it adequate for performing large-scale numerical analysis while still counting with a significant level of information for each material at each integration point of the structure. This does not limit nor influence in any way the type of loading that can be applied and therefore paves the way for the simulation of complex loading combinations that include self-weight, concentrated loads, pressure loads, pre-stressing loads, etc.

In this sense, this paper shows that the S-P RoM coupled with the imposed strain procedure is a valuable tool for the case of advanced nonlinear behaviour in both components of a composite material.

Two different cases have been shown, of two different structures with varying levels of complexity. They serve as an example of industrial application on real life structures that can be commonly found in the field of civil engineering. Their structural performance is analysed in detail in the nonlinear domain and both the evolution of the concrete and the steel is shown by means of damage and plasticity models.

A new crack opening metric is also proposed for the concrete in order to achieve a better understanding of the effect of high loading levels and advanced degradation in the material from a mascroscale point of view.

The examples shown also deal with different tendon trajectories thru the concrete structure and therefore serve as a demonstration that the methodology proposed can be applied to any steel trajectory, independent of its curvature.

We can therefore conclude that the formulation is capable of simulating the effect of the pre-stressing of tendons in any type of structure with any shape and/or boundary condition in the advanced nonlinear domain.

Acknowledgements: This work has been partially supported by the Spanish "Ministerio de Economía y Competividad" through the projects BIA2015-67807-R - RESCICLO and EUIN2015-62818 - RESSAFE and by the European Commission by means of the project TCAiNMaND- PIRSES-GA-2013612607.

\section{References}

[1] Rastellini, F., Oller, S., Salomon, O., Oñate, E. Composite materials non-linear modelling for long fibre reinforced laminates: Continuum basis, computational aspects and validations. Computers and Structures 2008; 86(9): 879-896.

[2] Martinez, X., Oller, S., Rastellini, F. and Barbat, A.H. A numerical procedure simulating RC structures reinforced with FRP using the serial/parallel mixing theory, Computers and Structures 2008; 86(1516):1604-18.

[3] Martinez, X., Oller, S., Rastellini, F. Análisis no-lineal de materiales compuestos mediante la teoría de mezclas serie-paralelo. Omnia Publisher, 2014. Capítulo 10 - p. 237-260. Libro: Aplicaciones avanzadas de los materiales compuestos en la obra civil y la edificación, (http://dx.doi.org/10.3926/oms.209)

[4] Cornejo, A., Barbu, L., Escudero, C., Martinez, X. , Oller, S. and Barbat, A. H., Methodology for the analysis of post-tensioned structures using a constitutive serial-parallel rule of mixtures, Composite Structures 2018; 200: 480-497. 
[5] Rabczuk, T., Eibl, J. Numerical analysis of prestressed concrete beams using a coupled element free Galerkin/finite element approach Internat J Solids Structures 2004; 41:1061-1080.

[6] Ayoub, A. , Filippou, F. Finite-element model for pretensioned prestressed concrete girders J Struct Eng 2010; 41: 401-409.

[7] Bílý, P., Kohoutková, A. A Numerical Analysis of the Stress-strain Behavior of Anchorage Elements and Steel Liner of a Prestressed Concrete Containment Wall, Structures 2017; 12: 24-39.

[8] Bílý, P., Kohoutková, A., An estimation of the effect of steel liner on the ultimate bearing capacity of prestressed concrete containment, Nuclear Engineering and Design 2018; 328: 197-208.

[9] Barbu, L., Escudero-Torres, C., Cornejo, A. , Martinez, X. , Oller, S. and Barbat, A. H., Analysis of pre-tensioned structures by means of a constitutive Serial-Parallel rule of mixtures, In: XIV International Conference on Computational Plasticity, Proceedings of the XIV International Conference on Computational Plasticity (Complas 2017), Barcelona, 5-7 September 2017. p. 471-482.

[10] Spanish Minister of Public Works. Instrucción de Hormigón Estructural EHE-08 (Spanish Structural Concrete Code); 2008.

[11] Kachanov, L. M. Time to the rupture process under creep conditions, Izv. Akad. Nauk. SSR Otd. Tech. Nauk. 1958; 8:26-31.

[12] Barbu, L.G., Oller, S., Martinez, X. and Barbat, A. High cycle fatigue simulation: A new stepwise load-advancing strategy, Engineering $\quad$ Structures $\quad 2015 ; \quad$ 97: http://dx.doi.org/10.1016/j.engstruct.2015.04.012.

[13] Oliver, J., Cervera, M., Oller, S., and Lubliner, J. Isotropic damage models and smeared crack analysis of concrete, In: Second international conference on computer aided analysis and design of concrete structures 1990; 2:945-58.

[14] Oller, S. Nonlinear dynamics of structures. Springer, Barcelona, Spain 2014. ISBN 978-3-319-05193-2

[15]Lubliner, J., Oliver, J., Oller, S. and Oñate, E., A plastic-damage model for concrete. Int. J. Solids Structures 1989; 25(3): 299-326.

[16] Martinez, X., Oller, S., Barbu, L., Barbat, A. and De Jesus, A.M.P., Analysis of ultra-low cycle fatigue problems with the Barcelona plastic damage model and a new isotropic hardening law, Int. J. Fatigue 2015; 73:132-142.

[17] Barbu, L.G., Martinez, X., Oller, S. and Barbat, A.H. Validation on large scale tests of a new hardening-softening law for the Barcelona plastic damage model, International Journal of Fatigue 2015; 81:213-226, doi:10.1016/j.ijfatigue.2015.07.031

[18] PLCd Manual. Non-linear thermo-mechanic finite element code oriented to PhD student education. Code developed at CIMNE; 1991-to present. URL: <http://www.cimne.com/plcd>. 